\title{
Incidence of Complications from Percutaneous Biopsy in Chronic Liver Disease: A Systematic Review and Meta-Analysis
}

\author{
Helena B. Thomaides-Brears ${ }^{1}$ (D) Naim Alkhouri ${ }^{2} \cdot$ Daniela Allende $^{3,4} \cdot$ Mukesh Harisinghani $^{5} \cdot$ Mazen Noureddin $^{6}$. \\ Nancy S. Reau ${ }^{7} \cdot$ Marika French $^{1}$ - Carlos Pantoja ${ }^{8} \cdot$ Sofia Mouchti ${ }^{1}$ - Donna R. H. Cryer ${ }^{4}$
}

Received: 24 December 2020 / Accepted: 31 May 2021 / Published online: 15 June 2021

(c) The Author(s) 2021

\begin{abstract}
Background Approaches to liver biopsy have changed over the past decade in patients with chronic liver disease.

Aims We conducted a systematic review and meta-analysis on the incidence of all complications and technical failure associated with percutaneous liver biopsy.

Methods We systematically searched PubMed and the Cochrane Library for cohort studies reporting on complications resulting from liver biopsy published between 2010 and 2020. Studies on participants of any age and sex, who underwent any percutaneous biopsy for non-focal liver disease, were selected. All events except mild pain, minor hematoma, vasovagal episodes, fever and fistula were defined as major complications. Random-effect model meta-analyses with and without covariates were performed, to examine the effect of publication year, patient characteristics, outcome collection, and biopsy type on incidences.

Results We identified 30 studies reporting on complications resulting from percutaneous liver biopsy procedures $(n=64,356)$. Incidence of major complications was $2.44 \%$ (95\% CI 0.85, 6.75), with mortality at $0.01 \%$ (95\% CI 0.00, 0.11), hospitalization at $0.65 \%$ (95\% CI $0.38,1.11)$, major bleeding at $0.48 \%$ (95\% CI 0.22, 1.06), and moderate/severe pain at $0.34 \%(95 \%$ CI $0.08,1.37)$. Minor complications at $9.53 \%(95 \%$ CI 3.68, 22.5) were mainly pain at $12.9 \%$ (95\% CI 5.34, 27.9). Technical failure was high at $0.91 \%(95 \% \mathrm{CI} 0.27,3.00)$. Decreasing patient age significantly increased incidence of hospitalization and major bleeding $(P<0.0001)$. Hospitalization incidence also significantly increased with disease severity.

Conclusions Incidence of major (2.4\%) and minor (9.5\%) complications, and technical failure $(0.91 \%)$ in percutaneous liver biopsies continues.
\end{abstract}

Keywords Percutaneous liver biopsy $\cdot$ Bleeding $\cdot$ Hospitalization $\cdot$ Pain

\section{Introduction}

Biopsy is considered the diagnostic reference for assessment of liver tissue in chronic liver disease. As outlined in 2009 by the American Association for the Study of Liver Disease [1] and confirmed by the British Society of Gastroenterology [2], percutaneous biopsy is the most commonly performed procedure [3], with transjugular biopsies performed in patients more vulnerable to complications, and endoscopic biopsies emerging as a safe alternative. The use of imaging to guide needle positioning or to assist the procedure, by identifying ascites for example, has largely superseded blind

Helena B. Thomaides-Brears

helena.thomaides-brears@ perspectum.com

Extended author information available on the last page of the article procedures in most cases, particularly in pediatric patients [4]. Digitization of slides and the first steps in automation of histological reads have also been reported [5]. Not all facilities have ultrasound (US), computer tomography (CT) or digitization, however, and real-world practice varies widely based on local guidelines.

Assessment of diffuse disease represents the most frequent biopsy indication, including for monitoring patients in drug efficacy trials. Over 2000 biopsies on average are performed per Phase 3 trial [6], as drug trial endpoints and for cohort enrichment, and this translates to $>50,000$ biopsies just in current active trials for fatty liver disease where no therapeutics have yet been licensed. For such disease a histological sample representative of the whole liver is required, but biopsy is subject to variability in sampling and interpretation [7-9]. 
Liver biopsy is invasive and is associated with potential clinical complications. These influence payer and healthcare costs so accurate estimates of complications are required to inform clinical guidelines. Clear contemporary incidences of such complications are lacking, however. Even now the quoted incidence of biopsy-related death, $0.01 \%$ [2], is based on a multi-center retrospective report in Italy [10] from over 30 years ago. Similarly, reported incidence of major bleeding varies widely, from $0 \%$ to $5.3 \%$, while pain incidence has been rarely reported on, but can reach $84 \%$ [11]. Very recently a scoping review on percutaneous biopsy has highlighted age, inpatient status, and coagulation profile as potential factors contributing to bleeding complications, which were estimated to be $<2 \%$ [3]. In contrast a metaanalysis of US-guided percutaneous liver biopsy for diffuse and focal disease reported $0.00 \%$ as the pooled incidence of bleeding. However, neither report fully examined all other complications [12].

Here our primary aim was to comprehensively evaluate recent incidence of every major and minor complication in real-world biopsy practice for chronic, non-focal liver disease. We aimed to standardize differences in reporting and examine the impact of risk factors on the incidence of complications. Due to their overwhelming prevalence, we focused on percutaneous liver biopsy procedures. The specific questions we sought to address were: (1) what are the current incidences of all reported complications, (2) are complications influenced by patient characteristics, biopsy procedure, and other study confounders, and (3) what is the technical failure rate of the procedure.

\section{Methods}

This systematic review was conducted and reported in accordance with the PRISMA (Preferred Reporting Items for Systematic Reviews and Meta-Analyses) statement [13].

\section{Literature Search}

We used free text terms for "liver" and "biopsy" and "complications" or "bleeding" to search the following electronic databases for abstracts published between January 2010 to April 2020: PubMed (including MEDLINE), Cochrane Library, ClinicalTrials.gov, WHO ICTRP including ISRCTN and EU Clinical Trials Register. The search results were combined into a Mendeley database to facilitate reference management. The bibliographic section of selected review articles, including both systematic and narrative articles on the topic, was manually searched for additional relevant articles (grey literature). The last search was performed on $4^{\text {th }}$ April 2020.

\section{Study Selection, Outcome and Covariate Definitions}

Studies were included in this review if they met the following criteria:

- Study design: prospective or retrospective human cohort studies including randomized control trials (RCTs).

- Population: pediatric (infant to 18 years) and/or adult ( $\geq 18$ years) patients with diffuse liver disease, including post-transplant and up to $25 \%$ focal disease.

- Exposure: any percutaneous liver biopsy (including US-guided, US-assisted, CT-guided, percussion-guided or plugged).

- Outcomes: within 3-months from biopsy, clearly reported counts for hospitalization, major and minor complications and technical failure.

Hospitalization events were study author defined serious adverse events and/or non-routine hospitalization events explicitly described, including transfusion. Major complications were defined by type of clinical event even if not associated with new or additional hospitalization or transfusion. Instead, the hospitalization category was used for events associated with new or additional hospitalization. Major complications comprised death, transfusion, major hematoma (subcapsular, intramuscular, intrahepatic, variceal), hemoperitoneum, hemothorax, hemobilia, organ injury, biliary event, pneumothorax, sepsis, moderate or severe pain (requiring follow-up, opiate analgesic or hospitalization, or $\geq 4$ on VAS scale). To address and include studies where major bleeding incidence was described without further specification of clinical diagnosis, the major bleeding category was introduced to include these bleeding events, as well as the specified major bleeding complications listed above (subcapsular hematoma, intramuscular hematoma, intrahepatic hematoma, variceal hematoma, hemoperitoneum, hemothorax, hemobilia).

Minor complications were mild pain (self-resolving, "at biopsy site" or $<4$ on VAS scale), other hematoma (localized at biopsy site, transthoracic, cervical), vasovagal episodes, fever, arteriovenous fistula. Technical failure was defined as an inability to attain a histological sample or achieve histological assessment.

To assess patient characteristics that may influence complication incidence studies with $\geq 25 \%$ patients with either ascites, transplants or cirrhosis were identified to create the covariate category of disease severity. Study covariates considered in the analysis were: publication year, cohort age (pediatric vs adult vs mixed age), severity of disease (chronic vs post-transplant vs cirrhosis vs ascites), outcome collection method (solicited vs 
spontaneous), biopsy procedure (percutaneous without or with US-guidance). In qualitative analysis, higher risk of bleeding was defined as $\geq$ INR 1.5 and/or platelet count below $50,000 / \mathrm{ml}$, although these are poor guides to bleeding tendency in those with cirrhosis [14].

We restricted inclusion to English language articles. We excluded studies reporting on biopsy in cohorts with hematopoietic stem cell transplant, sphincterotomy, transcatheter embolization or $>25 \%$ focal disease. We excluded studies reporting on transjugular, endoscopic biopsies or biopsies under direct visualization; although if clearly defined outcomes on percutaneous biopsies were included in the same article, the percutaneous subgroup was included as a separate study. If more than one study was reported by the same institute, only the most comprehensive was included.

\section{Data Extraction and Risk of Bias Assessment}

The following information was extracted from included studies independently by 2 co-authors and conflicts resolved by discussion: study design, participant characteristics, biopsy procedure details, outcomes reported, timing and method of follow-up, and outcome results. Study risk of bias was assessed through use of the QUIPS (QUality In Prognosis Studies) tool [15], modified based on the Newcastle-Ottawa scale criteria [16], and taking into account the current guidance on meta-analysis of cohort studies [17], to suit the requirements of this review (Supplementary Table 1). The modified tool has six domains: study population; study attrition; study procedure; study confounders; outcome measurement; and outcome reporting. For each domain, the adequacy of reporting by a study was assessed as "yes risk of bias," "partial," or "no risk of bias," by two co-authors. Based on domain assessments by two co-authors and following any conflict resolution, studies were assigned to the following overall categories of risk of bias:

- Low risk of bias: describes studies for which all domains had no risk;

- Moderate risk of bias: describes studies for which one or more domains are scored as "partial" or one domain is scored as "yes risk of bias";

- High risk of bias: describes studies for which more than one domain is scored as "yes risk of bias."

The rating of the overall quality of the evidence from this review was undertaken in consideration of current guidance on the GRADE (Grading of Recommendations, Assessment, Development and Evaluations) approach [18]. Sensitivity analysis was also carried out by separate meta-analysis of percutaneous biopsy complications in single-center studies and from all pediatric only studies.

\section{Statistical Analysis}

Data were analyzed in accordance with the Cochrane Handbook for Systematic Reviews of Interventions [17].

Only 4 studies reported on all complications, so for each analysis we included the subset with complete cases in the outcome and fitted random effects meta-analysis models to the data to derive pooled incidences and $95 \% \mathrm{CI}$ and sampling from a minimum of 6 studies. We assumed the incidence followed a binomial-normal distribution with a random intercept, as recommended for proportion-based analysis with low counts of events resulting in random dispersion of data based on the sample size [19]. Studies with no events were problematic and did not allow the calculation of study sampling variances. To address this, zero events were replaced with 0.5 , also increasing the total sample size by 1 . Statistical heterogeneity between studies was assessed using the likelihood ratio test [20] and Cochrane $I^{2}$ statistic cut-offs, with $50 \%$ to $90 \%$ potentially representing substantial heterogeneity and $75 \%$ to $100 \%$ representing considerable heterogeneity [17].

Forest plots were plotted to show the fitted random-effects model. Funnel plots of sample size by log odds were plotted to investigate the presence of publication bias or the effect of small study size by visual inspection [19].

The mixed effects model with study level covariates accounted for at least part of the heterogeneity in true effects and were explored for systematic differences between the studies. All study level covariates were transformed to the mean. This analysis was restricted to those outcomes with meaningful funnel plots. The aggregated influence of study level covariates on pooled incidences was tested via the $\mathrm{Q}$ Moderator-test (QM-test), while the influence of each covariate was also tested individually. Significance threshold of $P$-value tests was set at 0.05 , though true significance for covariate influence was set to $<0.001$.

All data analyses were conducted using $\mathrm{R}$ studio version 4.0.0.

\section{Results}

\section{Study Selection and Quality Assessment}

We screened a total of 5,973 studies and selected a total of 140 studies for full text evaluation. Following review of the full study articles, a total of 30 studies (28 articles), representing 64,356 percutaneous biopsies, were judged to meet the inclusion criteria for this systematic review and metaanalysis. The most common reasons for exclusion were the absence of biopsy complication numbers $(n=52)$ or studies that were qualitative reviews $(n=28)$ (Fig. 1 , Supplementary Table 2). We excluded one study reporting on biopsy under 


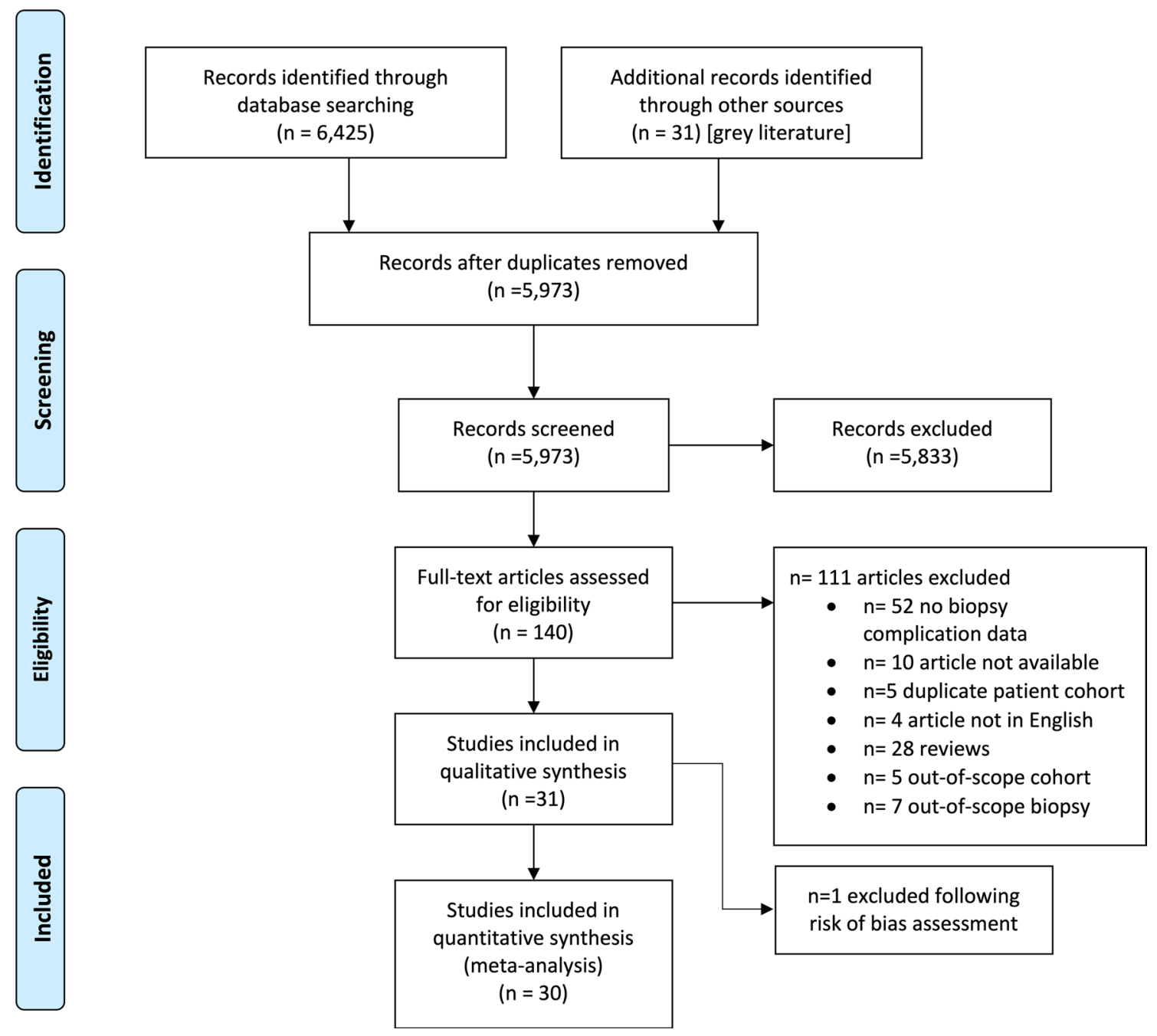

Fig. 1 PRISMA diagram summarizing study selection process

direct visualization, one study reporting on multiple procedure types without distinction, 3 studies with endoscopic and 4 studies on transjugular biopsies, as these studies were too few to enable valid meta-analysis. We also excluded studies for which full articles not available $(n=10)$, if they were not in English $(n=4)$, they duplicated other studies $(n=5)$ or were out for scope $(n=5)$.

The QUIPS (QUality In Prognosis Studies) tool [15], modified based on the Newcastle-Ottawa scale criteria [16] and current guidance on cohort studies [17], were used to evaluate the quality of the studies. Most studies (17) were high quality, with 12 deemed to be of moderate quality and 1 judged to be of low quality (Supplementary Table 3).

\section{Study and Cohort Characteristics}

Most studies were retrospective (20/30, all except [21-29]). Most were single-center accounts, with 3 being multi-center studies that nevertheless represented the majority of samples (56,058 of 64,356 biopsies; Table 1). The overall study period represented was 1998-2017, with Asia-Pacific represented by 5 studies, Europe by 12, USA by 8 and Asia Minor by 5 studies.

Eighteen of 30 studies were performed exclusively on adults, while 4 were mixed age but mainly in adults. Eight studies were on pediatric patients and almost all utilized sedation (7/8). In contrast, sedation was reported in only 3 of 18 studies with adult-only patients. The majority of studies (18/30) were on less severe disease, while 10 included investigation of transplants (Supplementary Table 5). Half of the studies (15/30) utilized US to guide the percutaneous biopsy procedure. 


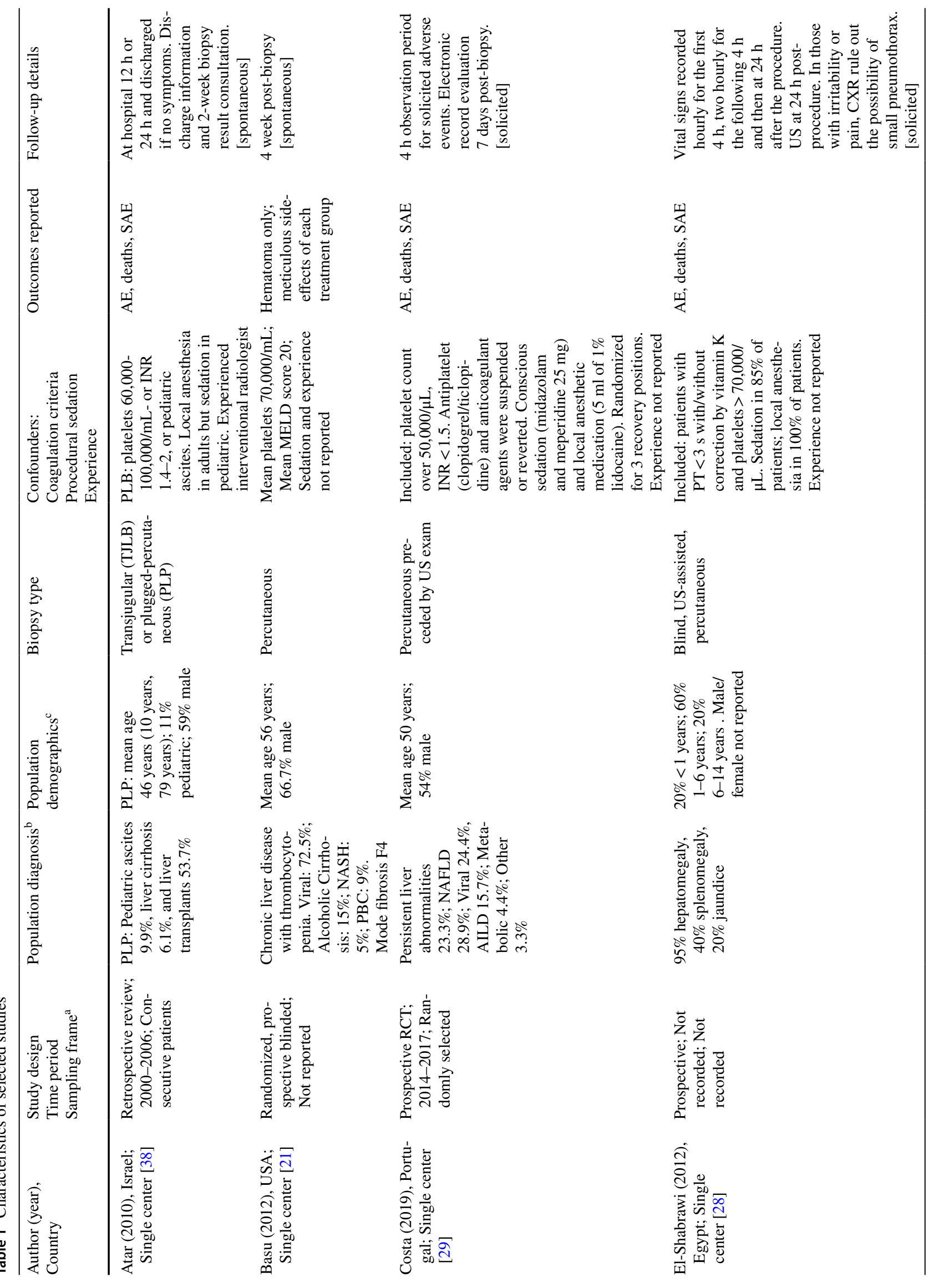




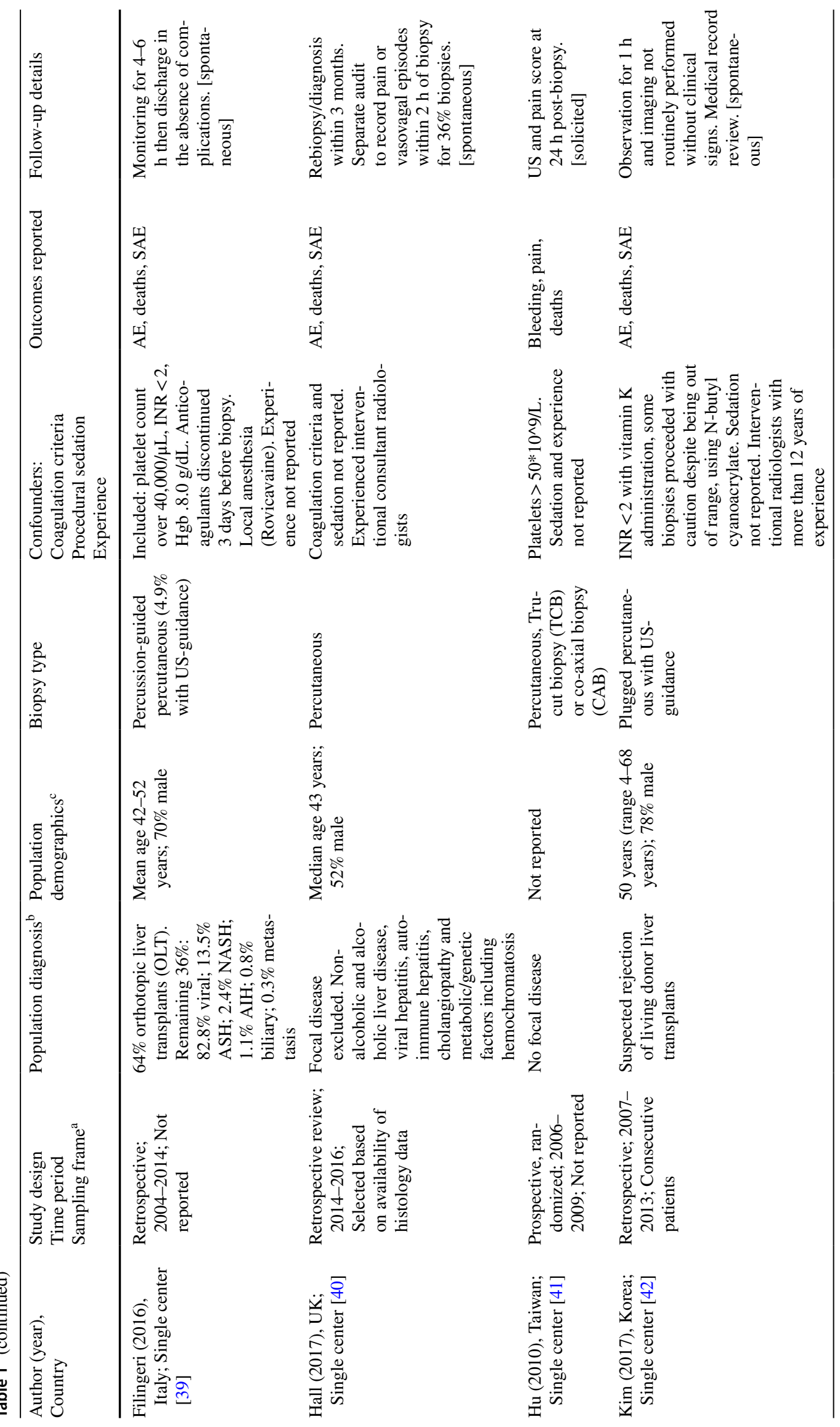




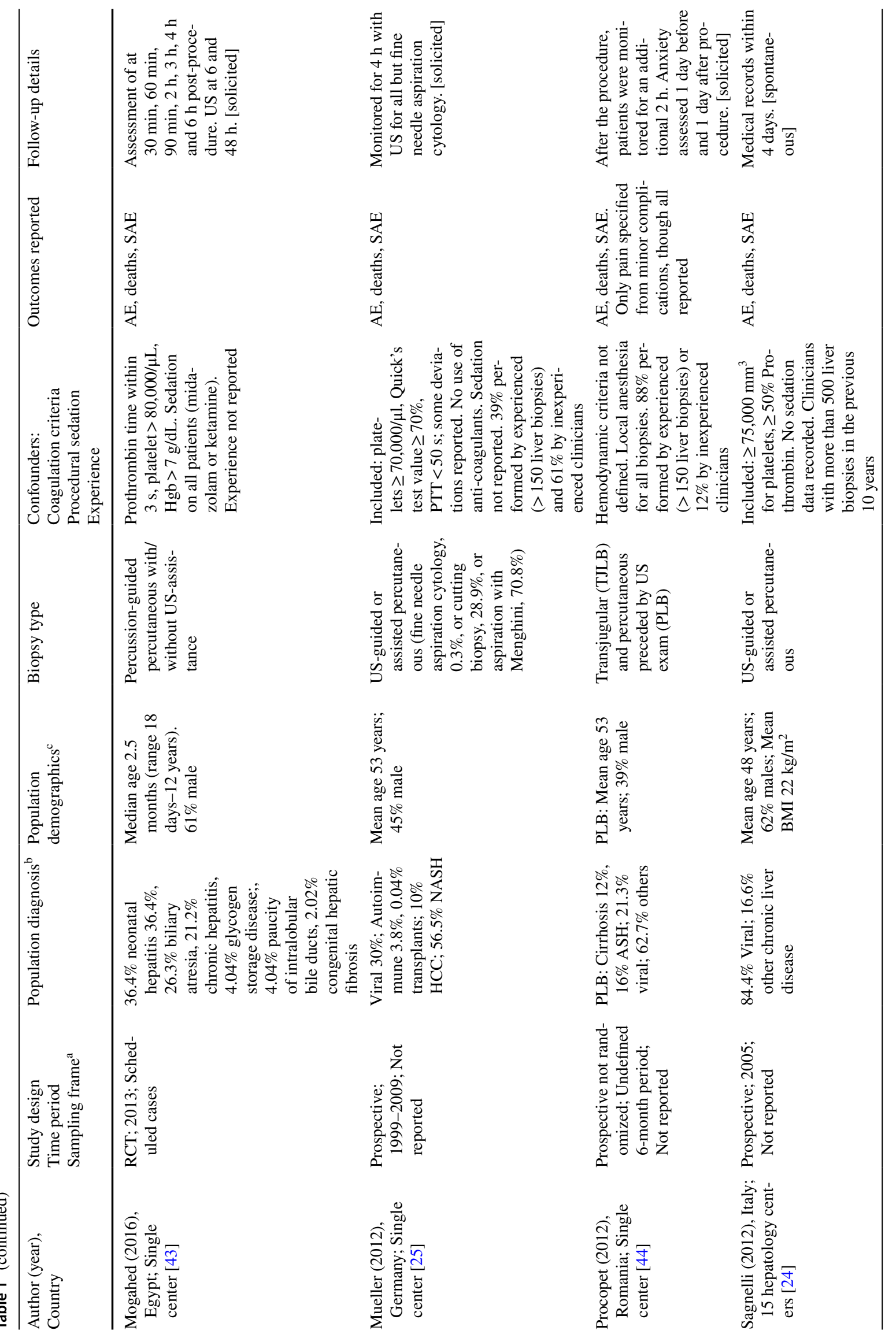




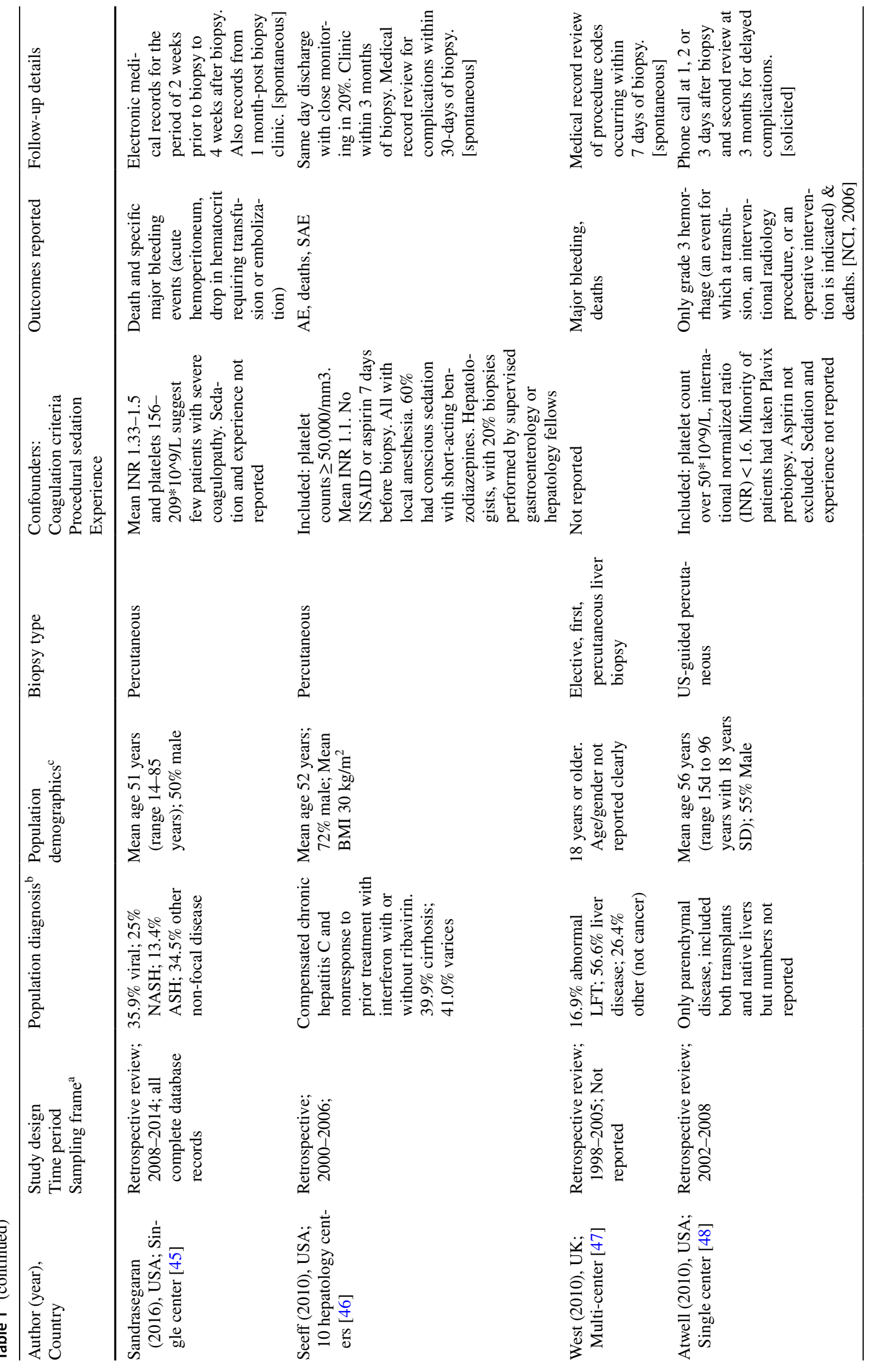




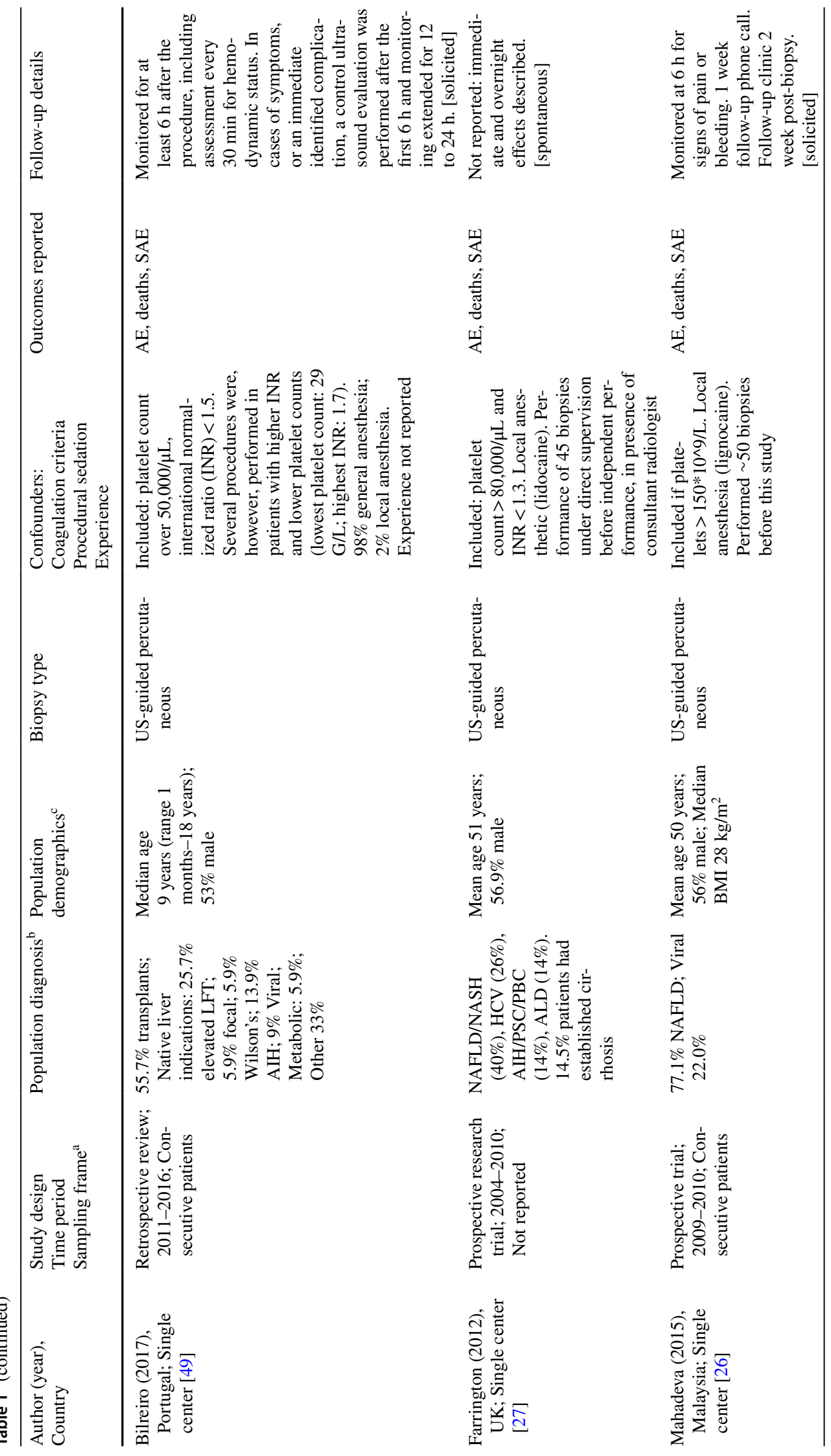




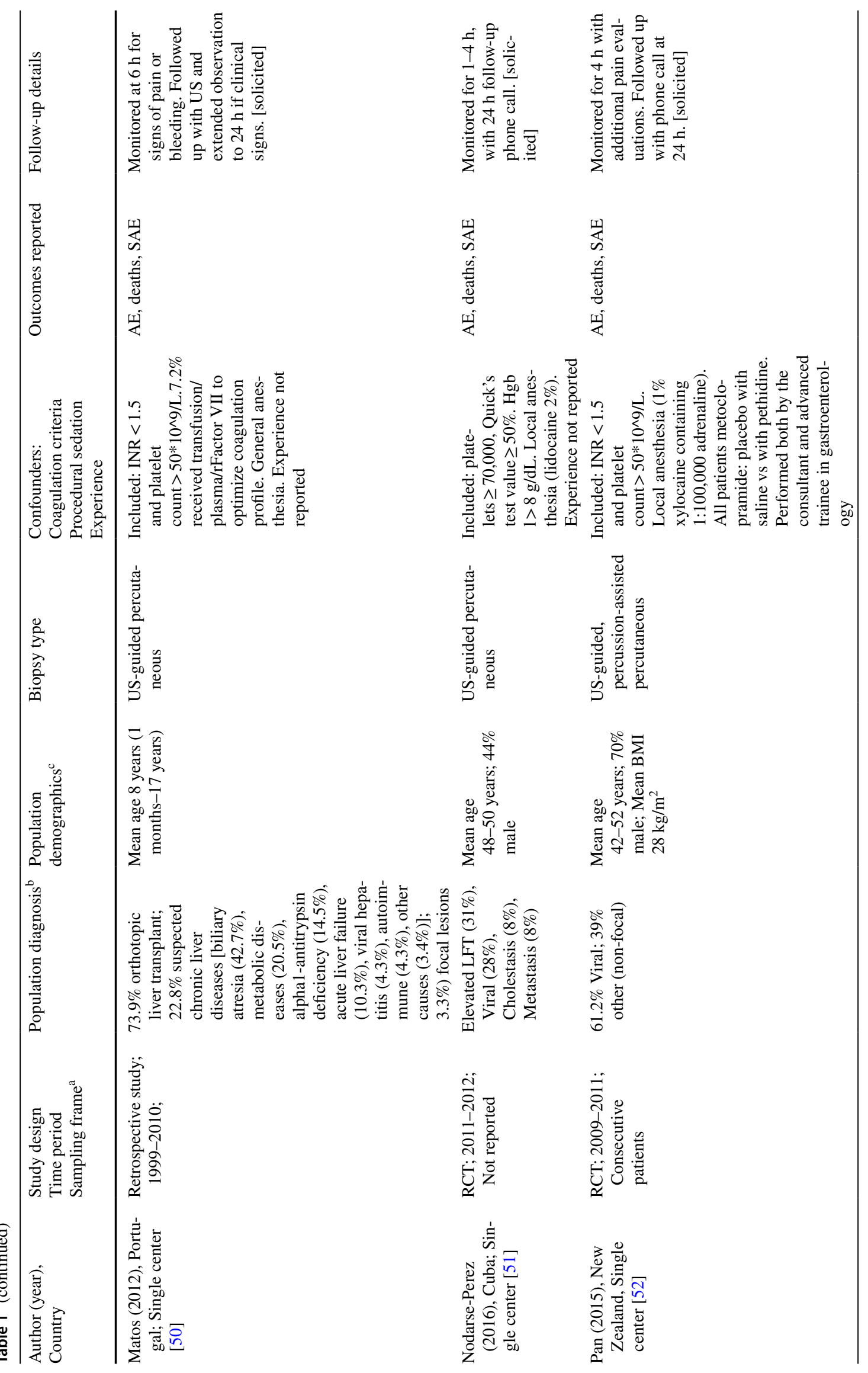




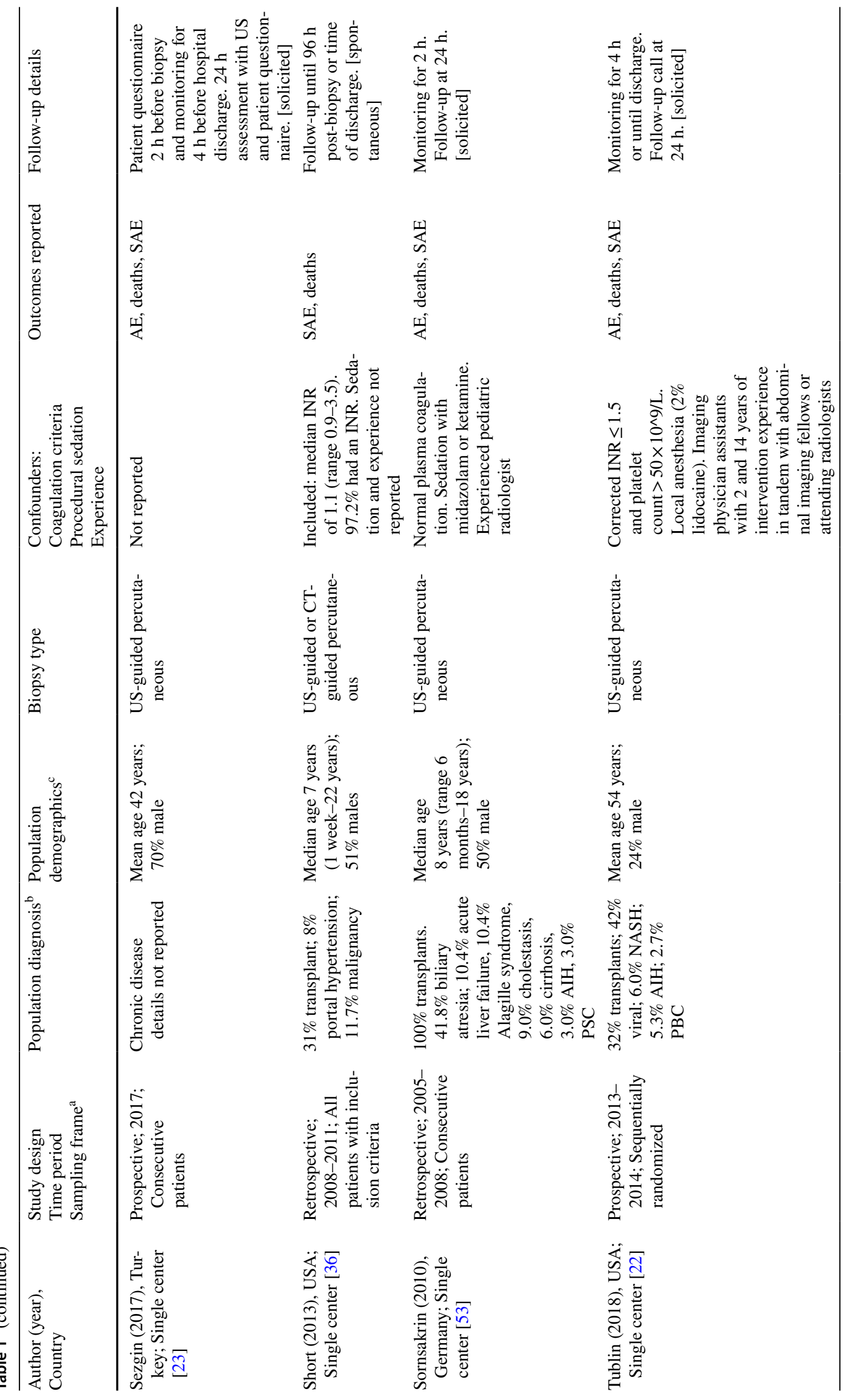




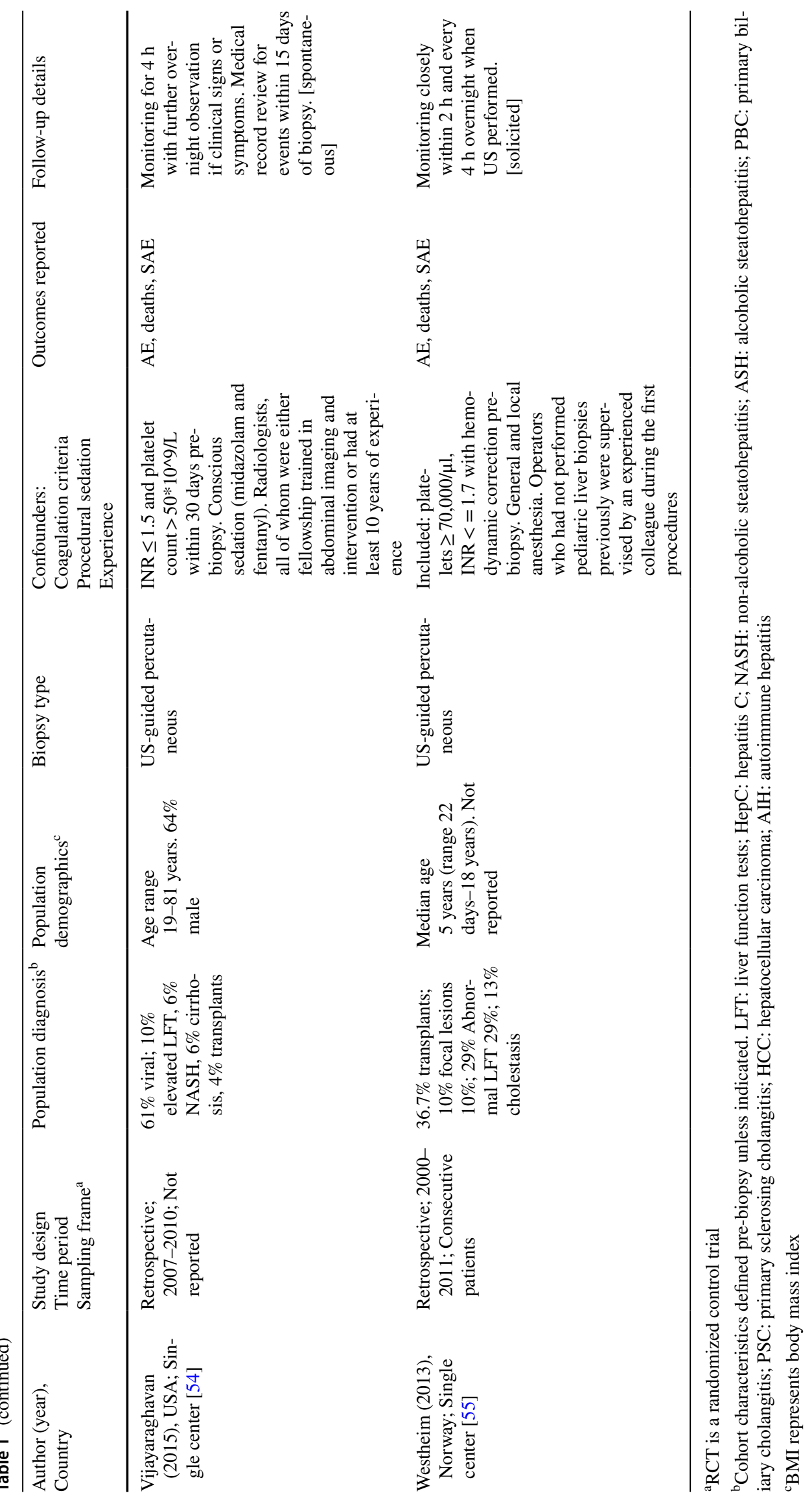


Table 2 Incidence of major complications, minor complications and technical failure

\begin{tabular}{|c|c|c|c|c|c|c|c|}
\hline Event & Pooled incidence $(95 \% \mathrm{CI})$ & $I^{2}(\%)$ & tau & $\begin{array}{l}\text { Heterogeneity } P \\
\text { value (LRT- } \\
\text { test) }\end{array}$ & $\begin{array}{l}\text { Included } \\
\text { studies }\end{array}$ & $\begin{array}{l}\text { Total events/ } \\
\text { Total biop- } \\
\text { sies }\end{array}$ & $\begin{array}{l}\text { By population age }{ }^{\text {b }} \\
\text { Total events/total biopsies }\end{array}$ \\
\hline \multicolumn{8}{|l|}{ Major complications } \\
\hline Biliary events & $0.00 \%(0.00,0.79)$ & 81 & 2.69 & 0.7079 & 27 & 4/9341 & A: 0/7443; M: 0/306; $P: 4 / 1682$ \\
\hline Death & $0.01 \%(0.00,0.11)$ & 76 & 2.06 & 0.5157 & 29 & $12 / 64214$ & $\begin{array}{l}A: 9 / 60390 ; M: 0 / 2142 \\
\quad P: 3 / 1682\end{array}$ \\
\hline Hemobilia & $0.01 \%(0.00,0.54)$ & 38 & 1.02 & 0.9997 & 25 & $3 / 8797$ & A: $3 / 7137 ; M: 0 / 306 ; P: 0 / 1354$ \\
\hline Sepsis & $0.02 \%(0.00,0.51)$ & 24 & 0.77 & 0.9768 & 24 & $2 / 8608$ & $A ; 1 / 7159 ; M: 0 / 306 ; P: 1 / 1143$ \\
\hline Hemothorax & $0.03 \%(0.01,0.10)$ & 0 & 0.00 & 0.9995 & 26 & $3 / 9125$ & $A: 2 / 7137 ; M: 0 / 306 ; P: 1 / 1682$ \\
\hline Organ perforation & $0.03 \%(0.01,0.10)$ & 0 & 0.00 & 0.9999 & 26 & 3/9013 & A: $2 / 7353 ; M: 0 / 306 ; P: 1 / 1354$ \\
\hline Pneumothorax & $0.04 \%(0.02,0.11)$ & 0 & 0.00 & 0.9999 & 27 & $4 / 9341$ & $A: 4 / 7353 ; M ; 0 / 306 ; P: 0 / 1682$ \\
\hline Hemoperitoneum & $0.06 \%(0.01,0.39)$ & 84 & 2.05 & 0.0002 & 26 & $27 / 9125$ & $\begin{array}{l}\text { A: 15/7137; M: 0/306; P: } \\
\quad \text { 12/1682 }\end{array}$ \\
\hline Transfusion & $0.08 \%(0.02,0.41)$ & 80 & 1.96 & $<0.0001$ & 25 & $20 / 8057$ & $\begin{array}{l}\text { A: } 7 / 6582 ; M 0 / 306 ; P: \\
\quad 13 / 1169\end{array}$ \\
\hline Major hematoma $^{c}$ & $0.11 \%(0.02,0.53)$ & 90 & 2.33 & $<0.0001$ & 25 & $39 / 8797$ & $\begin{array}{l}\text { A: } 14 / 7137 ; M: 0 / 306 ; P: \\
\quad 25 / 1354\end{array}$ \\
\hline Moderate/severe pain & $0.34 \%(0.08,1.37)$ & 96 & 2.41 & $<0.0001$ & 21 & $132 / 8346$ & $A: 112 / 7012 ; P: 20 / 1334$ \\
\hline Major bleeding & $0.48 \%(0.22,1.06)$ & 94 & 1.58 & $<0.0001$ & 30 & $356 / 64356$ & $\begin{array}{l}\text { A: 293/60390; } M: 14 / 2284 ; P: \\
\quad 49 / 1682\end{array}$ \\
\hline Hospitalization & $0.65 \%(0.38,1.11)$ & 86 & 1.01 & $<0.0001$ & 30 & $361 / 64356$ & $\begin{array}{l}\text { A: } 318 / 60390 ; M: 14 / 2284 ; P: \\
\quad 29 / 1682\end{array}$ \\
\hline All major complications & $2.44 \%(0.85,6.75)$ & 97 & 1.86 & $<0.0001$ & 15 & $184 / 6013$ & $A: 153 / 5831 ; P: 31 / 182$ \\
\hline Technical failure & $0.91 \%(0.27,3.00)$ & 97 & 2.17 & $<0.0001$ & 17 & $166 / 6801$ & $\begin{array}{l}\text { A: } 90 / 4893 ; M: 5 / 306 ; P \text { : } \\
\quad 71 / 1602\end{array}$ \\
\hline \multicolumn{8}{|l|}{ Minor complications } \\
\hline Vasovagal episodes & $0.15 \%(0.03,0.66)$ & 89 & 2.28 & $<0.0001$ & 23 & 34/7781 & $\begin{array}{l}\text { A: } 33 / 6432 ; M: 0 / 306 ; P: \\
\quad 1 / 1043\end{array}$ \\
\hline Other hematoma ${ }^{\mathrm{d}}$ & $0.19 \%(0.03,1.14)$ & 97 & 2.55 & $<0.0001$ & 15 & $80 / 7177$ & $A: 10 / 5925 ; P: 70 / 1252$ \\
\hline Other minor events ${ }^{\mathrm{e}}$ & $0.27 \%(0.07,1.03)$ & 94 & 2.51 & $<0.0001$ & 23 & $65 / 8265$ & $\begin{array}{l}A: 29 / 6277 ; M: 1 / 306 ; P: \\
\quad 35 / 1682\end{array}$ \\
\hline Mild pain & $12.9 \%(5.34,27.9)$ & 99 & 2.10 & 0.7079 & 19 & $704 / 7247$ & A: $645 / 6241 ; P: 59 / 1006$ \\
\hline All minor events & $9.53 \%(3.68,22.5)$ & 99 & 1.69 & $<0.0001$ & 11 & $424 / 6242$ & $A: 381 / 5649 ; P: 43 / 593$ \\
\hline
\end{tabular}

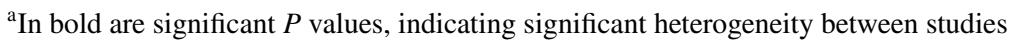

${ }^{\mathrm{b}} \mathrm{A}$ : adults; M: mixed age group; P: pediatric patients

${ }^{\mathrm{c}}$ Subcapsular, intramuscular, intrahepatic, variceal hematoma

${ }^{\mathrm{d}}$ Hematoma at biopsy site, transthoracic, cervical hematoma

${ }^{\mathrm{e}}$ Arteriovenous fistula, fever, other (not pain or vasovagal episodes)

\section{Major Complications}

The pooled incidence of major complications for percutaneous biopsies was $2.44 \%$ (95\% CI 0.85, 6.75) (Table 2; Figs, $2,3,4,5,6,7,8)$. Mortality occurred at $0.01 \%(95 \% \mathrm{CI}$ $0.00,0.11)$, major bleeding at $0.48 \%$ (95\% CI $0.22,1.06)$ and moderate or severe pain at $0.34 \%$ (95\% CI $0.08,1.37)$. Incidence of non-routine hospitalization was $0.65 \%$ (95\% CI $0.38,1.11)$. Other specific complications occurred rarely, ranging from $0 \%(95 \%$ CI $0.00,0.79)$ for biliary events and $0.11 \%$ (95\% CI $0.02,0.53$ ) for major hematomas (subcapsular, intramuscular, intrahepatic or variceal).
While not a major complication, incidence of technical failure during biopsy procedures has significant consequences for both patient and clinician. Technical failure occurred at $0.91 \%$ (95\% CI of $0.27,3.00$ ), based either on the failure to attain a histological sample at all or a sample without sufficient quality for scoring (Fig. 9). Potential publication bias was investigated with funnel plots (Supplementary Figs. 1-2) and identified the absence of bias for hospitalization, major complications, moderate/severe pain and technical failure. 
A

Study

Total N. event

Estimate $[95 \% \mathrm{Cl}]$

$\begin{array}{lcc}\text { Sandrasegaran, } & 142 & 7 \\ \text { Costa, } & 90 & 0 \\ \text { El-Shabrawi , } & 80 & 0 \\ \text { Hu, } & 276 & 0 \\ \text { Kim, } & 73 & 0 \\ \text { Mogahed, } & 57 & 6 \\ \text { Mogahed, } & 45 & 1 \\ \text { Procopet, } & 68 & 1 \\ \text { Atar, } & 233 & 0 \\ \text { Atwell, } & 1836 & 7 \\ \text { Bilreiro, } & 228 & 0 \\ \text { Farrington, } & 216 & 2 \\ \text { Filingeri, } & 1011 & 15 \\ \text { Hall, } & 194 & 0 \\ \text { Mahadeva, } & 203 & 0 \\ \text { Matos, } & 513 & 5 \\ \text { Mueller, } & 1015 & 5 \\ \text { Nodarse-Perez, } & 85 & 1 \\ \text { Pan, } & 48 & 0 \\ \text { Pan, } & 50 & 0 \\ \text { Sagnelli, } & 281 & 1 \\ \text { Seef, } & 2740 & 29 \\ \text { Sezgin, } & 90 & 0 \\ \text { Short, } & 328 & 11 \\ \text { Sornsakrin, } & 120 & 2 \\ \text { Tublin, } & 150 & 1 \\ \text { Vijayaraghavan, } & 771 & 6 \\ \text { Westheim, } & 311 & 4 \\ \text { Basu, } & 65 & 0 \\ \text { West, } & 53037 & 257 \\ \text { Was } & & \end{array}$

$4.93[1.37,8.49]$

$0.55[0.00,2.07$.

$0.180 .00,0.68$

$0.68[0.00,2.54$

10.53 [2.56, 18.49]

$2.22[0.00,6.53]$

$1.470 .00,4.33$.

$0.210 .00,0.81$

$0.380 .10,0.66$

$0.930 .00,2.20$

$1.480 .74,2.23$

$0.260 .00,0.97$

$0.250 .00,0.92$.

$0.970 .12,1.82$

$0.490 .06,0.92$

$1.020 .00,3.83$

$0.980 .00,3.68$

$0.980 .00,3.68$

$0.360 .00,1.05$

$1.060 .68,1.44$

$0.55[0.00,2.07$.

$1.670 .00,3.96$

$0.670 .00,1.97$

$0.780 .16,1.40$

$1.29[0.03,2.54$

$\begin{array}{llll}0.76 & 0.00, & 2.85 \\ 0.48 & 0.43, & 0.54\end{array}$

$0.65[0.38,1.11]$

RE Model
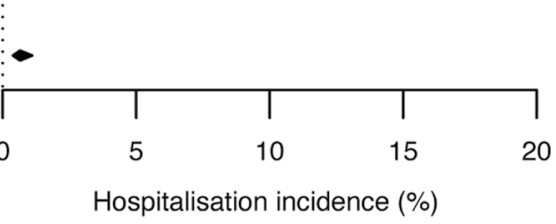

B

Study

Costa,

El-Shabrawi ,

Mogahed,

Mogahed,

Procopet,

Filingeri,

Mahadeva,

Mueller,

Nodarse-Perez,

Pan,

Pan,

Sagnelli,

Seef,

Sezgin,

Tublin,

RE Model
Total N. event

Estimate $[95 \% \mathrm{Cl}]$

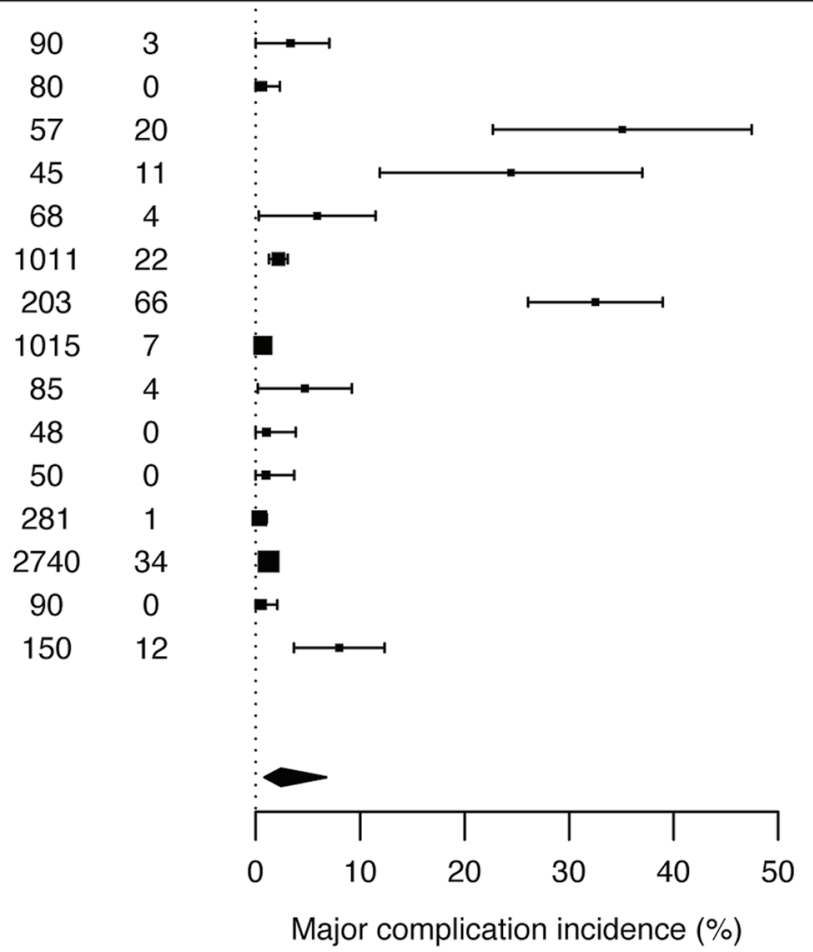

$3.33[0.00,7.04]$

$0.62[0.00,2.32]$

35.09 [22.70, 47.48]

24.44 [11.89, 37.00]

$5.88[0.29,11.47]$

2.18 [1.28, 3.08]

32.51 [26.07, 38.96]

$0.69[0.18,1.20]$

4.71 [0.20, 9.21]

$1.02[0.00,3.83]$

$0.98[0.00,3.68]$

$0.36[0.00,1.05]$

$1.24[0.83,1.66]$

$0.55[0.00,2.07]$

$8.00[3.66,12.34]$

$2.44[0.85,6.75]$

Fig. 2 Forest plots showing pooled incidence (as \%) for hospitalization (a) and all major complications (b) 
A

Study

Total N. event

Estimate $[95 \% \mathrm{Cl}]$

Sandrasegaran,

Costa,

El-Shabrawi

Hu,

Mogahed

Mogahed,

Procopet,

Atar,

Atwell,

Bilreiro,

Farrington,

Filingeri,

Hall,

Mahadeva

Matos,

Mueller,

Nodarse-Perez,

Pan,

Pan,

Sagnelli,

Seef,

Sezgin,

Short,

Sornsakrin,

Tublin,

Vijayaraghavan,

Westheim,

Basu,

West,

$\begin{array}{cc}142 & 7 \\ 90 & 0 \\ 80 & 0 \\ 276 & 0 \\ 73 & 0 \\ 57 & 9 \\ 45 & 5 \\ 68 & 1 \\ 233 & 0 \\ 1836 & 7 \\ 228 & 0 \\ 216 & 1 \\ 1011 & 8 \\ 194 & 0 \\ 203 & 0 \\ 513 & 8 \\ 1015 & 3 \\ 85 & 0 \\ 48 & 0 \\ 50 & 0 \\ 281 & 1 \\ 2740 & 16 \\ 90 & 0 \\ 328 & 11 \\ 120 & 1 \\ 150 & 0 \\ 771 & 6 \\ 311 & 15 \\ 65 & 0 \\ 53037 & 257 \\ & \end{array}$

$4.93[1.37,8.49$

$0.550 .00,2.07$

$0.620 .00,2.32$

$0.6800,254$

$15.79[6.32,25.26$

11.11 [1.93, 20.29

$1.47[0.00,4.33$

$0.210 .00,0.81$

$0.380 .10,0.66$

0.76 0.00, 1.37

$0.790 .25,1.34$

$\begin{array}{lll}0.26 & 0.00, & 0.97 \\ 0.25 & 0.00, & 0.92 \\ 1.56 & 0.49, & 2.63\end{array}$

$1.560 .49,2.63$

$0.30[0.00,0.63$

$0.580 .00,2.19$

$1.020 .00,3.83$

$\begin{array}{lll}0.98 & 0.00, & 3.68 \\ 0.36 & 0.00,1.05\end{array}$

$0.580 .30,0.87$

$0.550 .00,2.07$

$3.351 .41,5.30$

$0.830 .00,2.46$

$0.780 .16,1.40$

4.82 2.44, 7.20

$0.76[0.00,2.85$

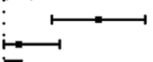

RE Model

$0.48[0.22,1.06]$

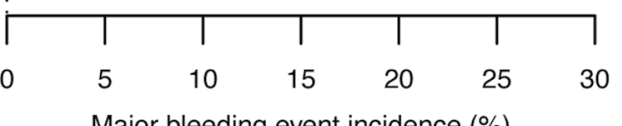

Major bleeding event incidence (\%)

B

Study

Total N. event

Estimate $[95 \% \mathrm{Cl}]$

$\begin{array}{lcc}\text { Costa, } & 90 & 3 \\ \text { El-Shabrawi , } & 80 & 0 \\ \text { Mogahed, } & 57 & 0 \\ \text { Mogahed, } & 45 & 0 \\ \text { Procopet, } & 68 & 1 \\ \text { Farrington, } & 216 & 2 \\ \text { Filingeri, } & 1011 & 5 \\ \text { Hall, } & 194 & 0 \\ \text { Mahadeva, } & 203 & 66 \\ \text { Matos, } & 513 & 0 \\ \text { Mueller, } & 1015 & 0 \\ \text { Nodarse-Perez, } & 85 & 3 \\ \text { Pan, } & 48 & 0 \\ \text { Pan, } & 50 & 0 \\ \text { Sagnelli, } & 281 & 0 \\ \text { Seef, } & 2740 & 7 \\ \text { Sezgin, } & 90 & 0 \\ \text { Short, } & 328 & 20 \\ \text { Tublin, } & 150 & 12 \\ \text { Vijayaraghavan, } & 771 & 13 \\ \text { Westheim, } & 311 & 0 \\ \end{array}$

RE Model

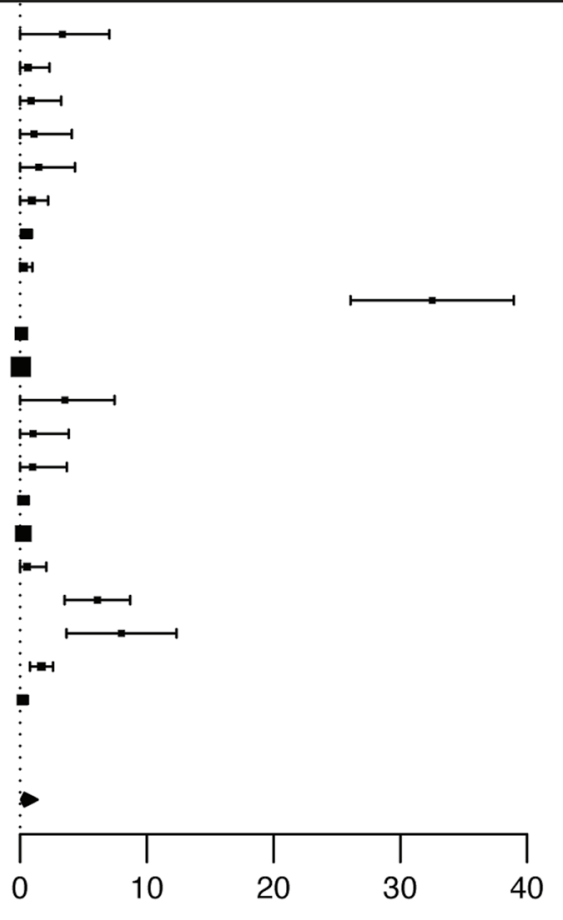

$3.33[0.00,7.04]$

$0.62[0.00,2.32]$

$0.86[0.00,3.24]$

$1.09[0.00,4.08]$

$1.47[0.00,4.33]$

$0.93[0.00,2.20]$

$0.49[0.06,0.93]$

$0.26[0.00,0.97]$

$32.51[26.07,38.96]$

$0.10[0.00,0.37]$

$0.05[0.00,0.19]$

$3.53[0.00,7.45]$

$1.02[0.00,3.83]$

$0.98[0.00,3.68]$

$0.18[0.00,0.67]$

$0.26[0.07,0.44]$

$0.55[0.00,2.07]$

$6.10[3.51,8.69]$

$8.00[3.66,12.34]$

$1.69[0.78,2.59]$

$0.16[0.00,0.60]$

$0.34[0.08,1.37]$

Moderate/severe pain incidence (\%)

Fig. 3 Forest plots showing pooled incidence (as \%) for major bleeding (a) and moderate/severe pain (b) 
A

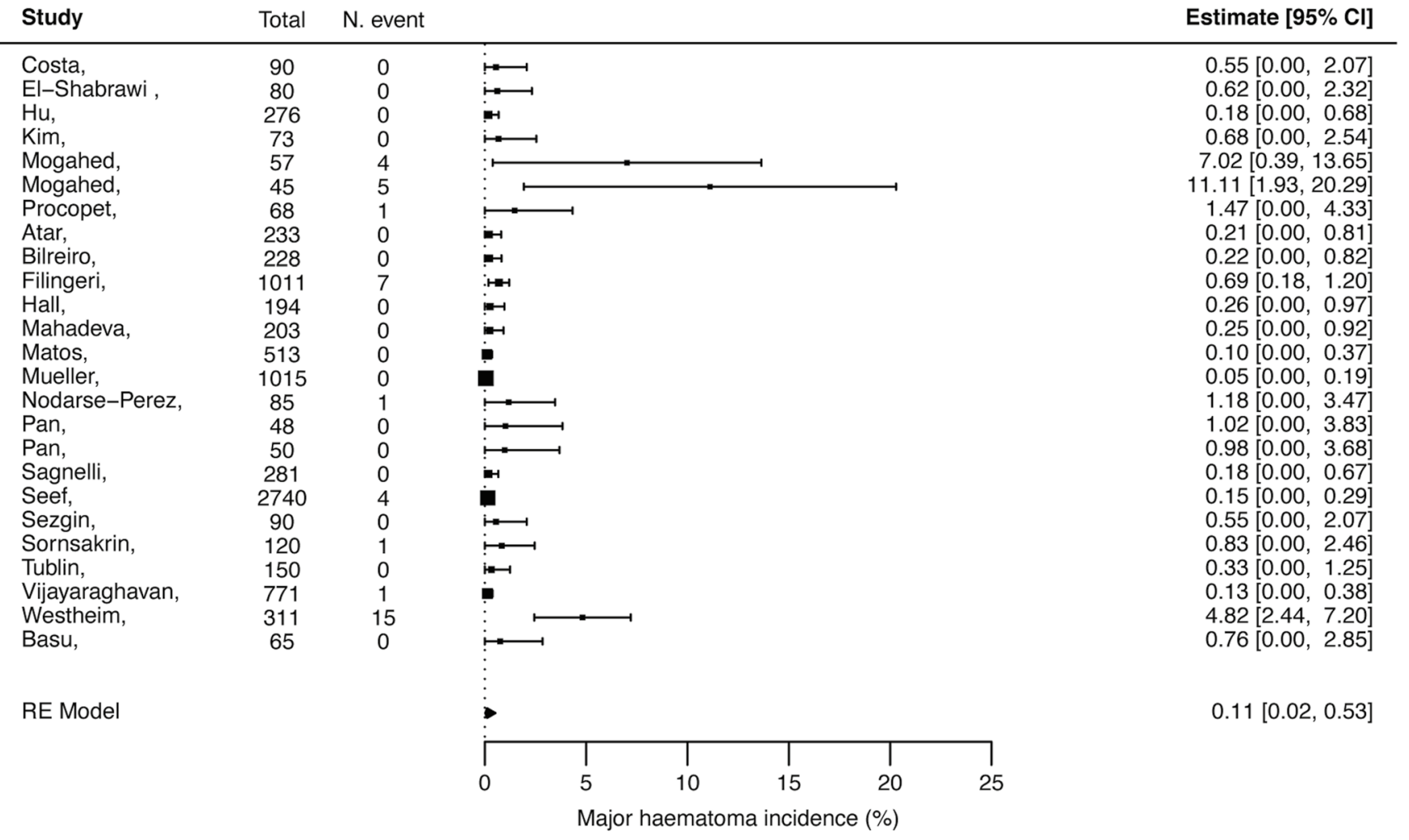

B

\begin{tabular}{lc} 
Study & Tota \\
\hline Costa, & 90 \\
El-Shabrawi, & 80 \\
Hu, & 276 \\
Kim, & 73 \\
Mogahed, & 57 \\
Mogahed, & 45 \\
Procopet, & 68 \\
Atar, & 233 \\
Bilreiro, & 228 \\
Filingeri, & 1011 \\
Hall, & 194 \\
Mahadeva, & 203 \\
Matos, & 513 \\
Mueller, & 1015 \\
Nodarse-Perez, & 85 \\
Pan, & 48 \\
Pan, & 50 \\
Sagnelli, & 281 \\
Seef, & 2740 \\
Sezgin, & 90 \\
Short, & 328 \\
Sornsakrin, & 120 \\
Tublin, & 150 \\
Vijayaraghavan, & 771 \\
Westheim, & 311 \\
Basu, & 65
\end{tabular}

RE Model

N. event

Estimate $[95 \% \mathrm{Cl}]$

$0.55[0.00,2.07]$

$0.62[0.00,2.32]$

$0.18[0.00,0.68]$

$0.68[0.00,2.54$

$7.02[0.39,13.65]$

$1.09[0.00,4.08$

$0.72[0.00,2.73]$

$0.21[0.00,0.81]$

$0.22[0.00,0.82$

$0.05[0.00,0.19]$

$0.26[0.00,0.97]$

$0.25[0.00,0.92]$

$1.56[0.49,2.63]$

$0.10[0.00,0.29]$

$0.58[0.00,2.19]$

$1.02[0.00,3.83$

$0.98[0.00,3.68]$

$0.36[0.00,1.05]$

$0.29[0.09,0.49]$

$0.55[0.00,2.07]$

$0.15[0.00,0.57]$

$0.41[0.00,1.56]$

$0.33[0.00,1.25$

$0.65[0.08,1.22]$

$0.16[0.00,0.60]$

$0.76[0.00,2.85]$

$0.06[0.01,0.39]$

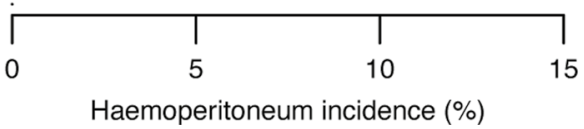

Fig. 4 Forest plots showing pooled incidence (as \%) for major hematoma (a) and hemoperitoneum (b) 
A

Stud

Total

N. event

Estimate $[95 \% \mathrm{Cl}]$

Costa,

$\mathrm{Hu}$,

Kim,

Mogahed

Mogahed,

Procopet,

Atar,

Atwell,

Farrington,

Filingeri,

Hall,

Mahadeva,

Matos,

Mueller,

Nodarse-Perez,

Pan,

Pan,

Sagnelli,

Seef,

Sezgin

Short,

Sornsakrin,

Tublin,

Vijayaraghavan,

Westheim,

Basu,

$\begin{array}{cc}90 & 0 \\ 80 & 0 \\ 276 & 0 \\ 73 & 0\end{array}$

0
0
0
0
2
0
0
0
0
0
0
0
0
0
0
0
0
0
0
0
0
0
1
0
0
1
0
0
8

$0.55[0.00,2.07]$

$0.62[0.00,2.32$

0.18 [0.00, 0.68

0.68 [0.00, 2.54]

$3.51[0.00,8.29$

$1.09[0.00,4.08$

$0.72[0.00,2.73$

$0.21[0.00,0.81$

0.03 [0.00, 0.10

$0.22[0.00,0.82$

0.23 [0.00, 0.87 ]

0.05 [0.00, 0.19

$0.26[0.00,0.97$

$0.25[0.00,0.92$

$0.10[0.00,0.37$

$0.05[0.00,0.19$

$0.58[0.00,2.19]$

$1.02[0.00,3.83$

$0.98[0.00,3.68$

$0.18[0.00,0.67]$

0.02 [0.00, 0.07

$0.55[0.00,2.07$

0.30 [0.00, 0.90]

$0.410 .00,1.56$

$0.33[0.00,1.25$

0.13 [0.00, 0.38

0.16 [0.00, 0.60

$0.76[0.00,2.85$

311
65

West,

53037

$-1$

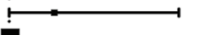

$\vdots$

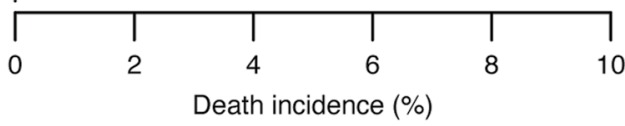

$0.01[0.00,0.11]$

B

Study

Total N. event

Estimate [95\% Cl]

\section{Costa,}

El-Shabrawi ,

$90 \quad 0$

$\mathrm{Hu}$,

276

Kim,

Mogahed,

Mogahed,

Procopet,

Atar,

Bilreiro,

Farrington,

Filingeri,

Hall,

Mahadeva,

Mueller,

Nodarse-Perez,

Pan,

Pan,

Sagnelli,

Seef,

Sezgin,

Short,

Sornsakrin,

Tublin,

Westheim,

Basu,

73
57

57

45
68

68
233

233

216

1011

194

203

1015
85

48
50

50

281

2740
90

90
328

120

150

311

65

RE Model

0
0
0
0
4
1
1
0
0
0
0
0
0
2
0
0
0
0
4
0
7
0
0
1
0

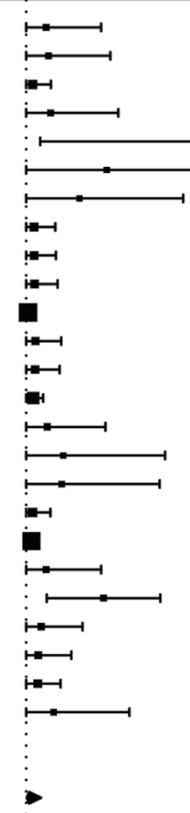

$0.55[0.00,2.07]$

$0.62[0.00,2.32]$

$0.18[0.00,0.68]$

$0.68[0.00,2.54$

$7.02[0.39,13.65]$

$2.22[0.00,6.53$

$1.47[0.00,4.33$

$0.21[0.00,0.81]$

$0.22[0.00,0.82$

$0.23[0.00,0.87$

$0.05[0.00,0.19]$

$0.26[0.00,0.97]$

$0.25[0.00,0.92$

$0.20[0.00,0.47]$

$0.58[0.00,2.19]$

$1.02[0.00,3.83]$

$0.98[0.00,3.68]$

$0.18[0.00,0.67]$

$0.15[0.00,0.29]$

$0.55[0.00,2.07]$

$2.13[0.57,3.70]$

$0.41[0.00,1.56]$

$0.33[0.00,1.25]$

$0.32[0.00,0.95]$

$0.76[0.00,2.85]$

$0.08[0.02,0.41]$

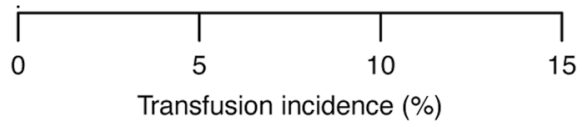

Fig. 5 Forest plots showing pooled incidence (as \%) for death (a) and transfusion (b) 
A

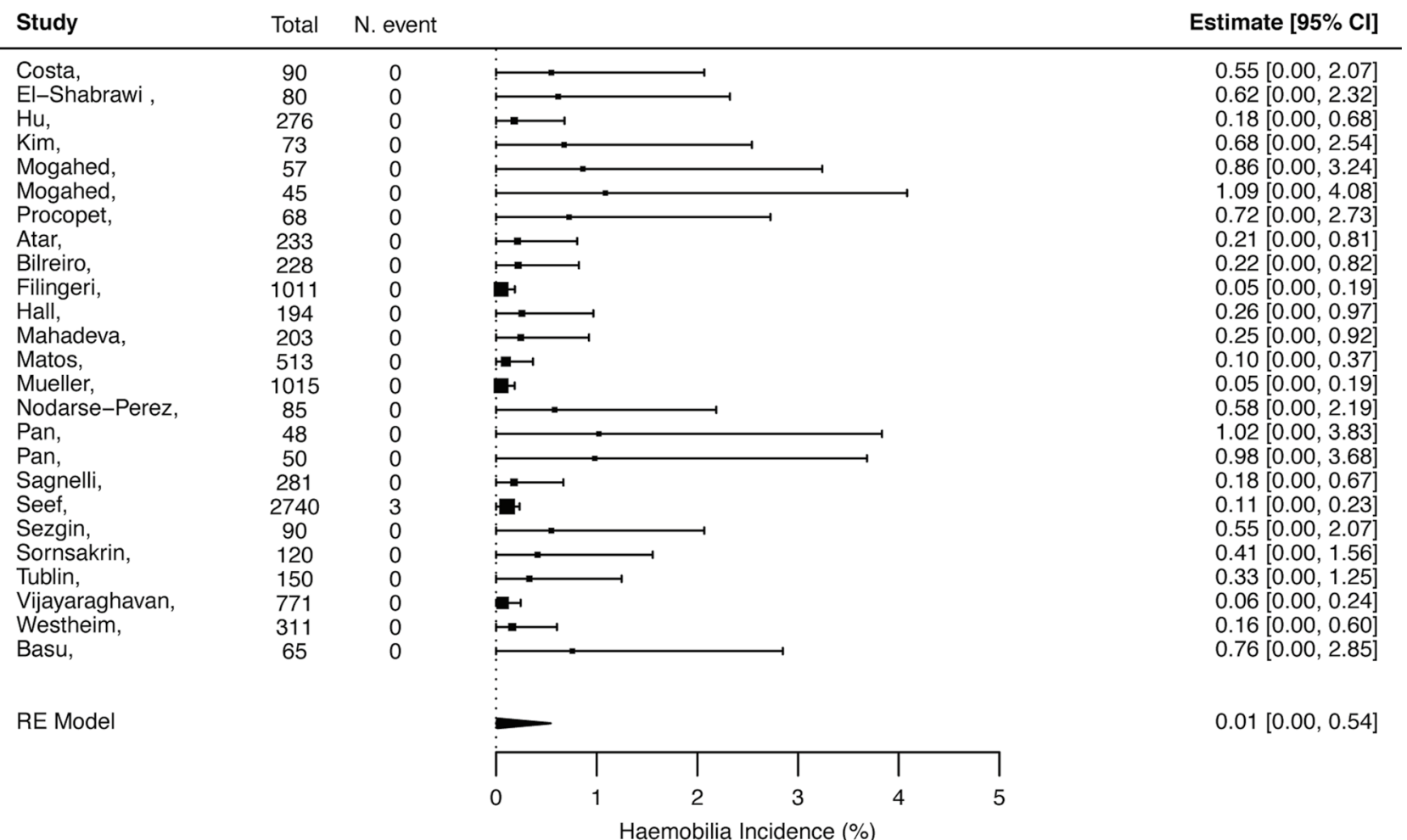

B

Study

Costa,

El-Shabrawi ,

$\mathrm{Hu}$,

Kim,

Mogahed,

Mogahed,

Procopet,

Atar,

Bilreiro,

Filingeri,

Hall,

Mahadeva,

Matos,

Mueller,

Nodarse-Perez,

Pan,

Pan,

Sagnelli,

Seef

Sezgin,

Short,

Sornsakrin,

Tublin,

Vijayaraghavan,

Westheim,

Basu,

RE Model
Total N. event

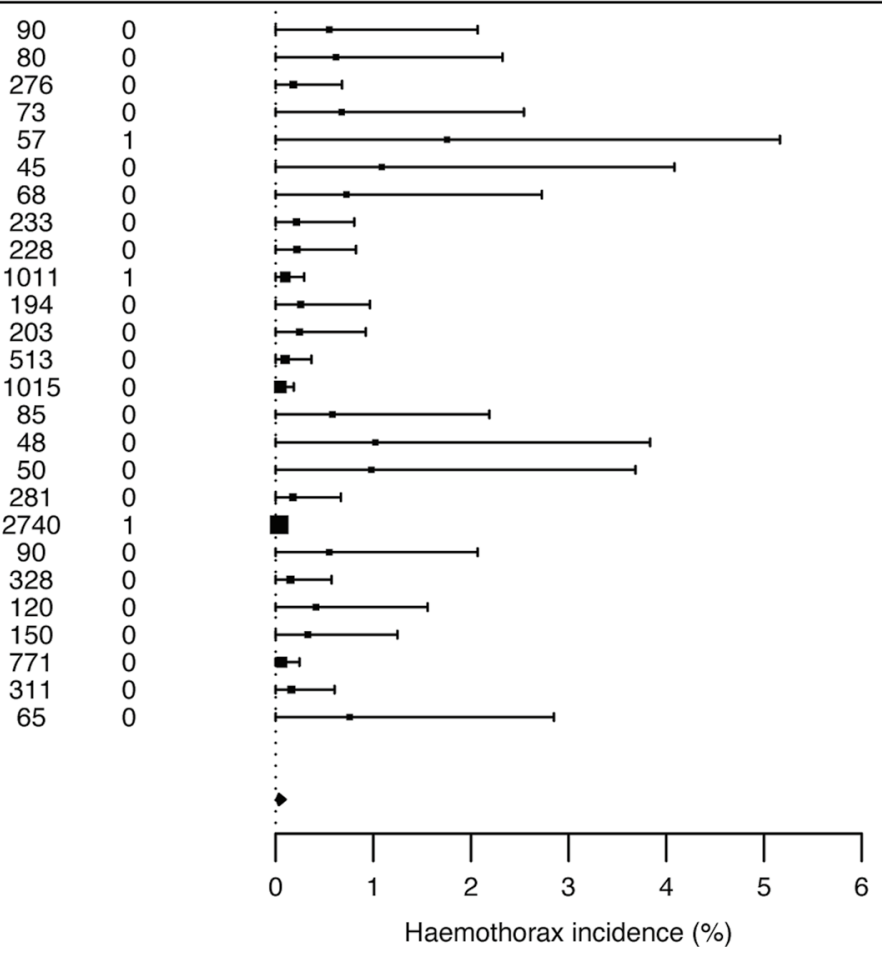

Estimate $[95 \% \mathrm{Cl}]$

$0.55[0.00,2.07]$

$0.62[0.00,2.32$

$0.18[0.00,0.68$

$0.68[0.00,2.54]$

$1.75[0.00,5.16$

$1.09[0.00,4.08$

$0.72[0.00,2.73$

$0.21[0.00,0.81$

$0.22[0.00,0.82$

$0.10[0.00,0.29$

$0.26[0.00,0.97$

0.25 [0.00, 0.92

$0.10[0.00,0.37$

$0.05[0.00,0.19$

$0.58[0.00,2.19$

$1.02[0.00,3.83$

$0.98[0.00,3.68$

$0.18[0.00,0.67$

$0.04[0.00,0.11]$

$0.55[0.00,2.07]$

$0.15[0.00,0.57$

$0.41[0.00,1.56$

$0.33[0.00,1.25]$

$0.06[0.00,0.24$

$0.16[0.00,0.60$

$0.76[0.00,2.85]$

$0.03[0.01,0.10]$

Fig. 6 Forest plots showing pooled incidence (as \%) for hemobilia (a) and hemothorax (b) 
A

Study

N. event Total

Estimate [95\% Cl]

Costa,

El-Shabrawi ,

$\mathrm{Hu}$,

Kim

Mogahed,

Mogahed,

Procopet,

Atar,

Bilreiro,

Farrington,

Filingeri,

Hall,

Mahadeva,

Matos,

Mueller,

Nodarse-Perez,

Pan,

Pan,

Sagnelli,

Seef,

Sezgin,

Short,

Sornsakrin,

Tublin,

Vijayaraghavan,

Westheim,

Basu,

$\begin{array}{ll}0 & 90 \\ 0 & 80 \\ 0 & 276 \\ 0 & 73 \\ 0 & 57 \\ 0 & 45 \\ 0 & 68 \\ 0 & 233 \\ 0 & 228 \\ 0 & 216 \\ 2 & 101 \\ 0 & 194 \\ 0 & 203 \\ 0 & 513 \\ 1 & 1015 \\ 0 & 85 \\ 0 & 48 \\ 0 & 50 \\ 0 & 281 \\ 1 & 274 \\ 0 & 90 \\ 0 & 328 \\ 0 & 120 \\ 0 & 150 \\ 0 & 771 \\ 0 & 311 \\ 0 & 65\end{array}$

RE Model

90
80
276
73
57
45
68
233
228
216
1011
194
203
513
1015
85
48
50
281
2740
90
328
120
150
771
311
65
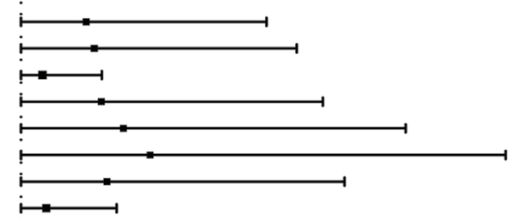

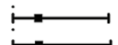
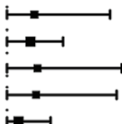

$+1$

!e-
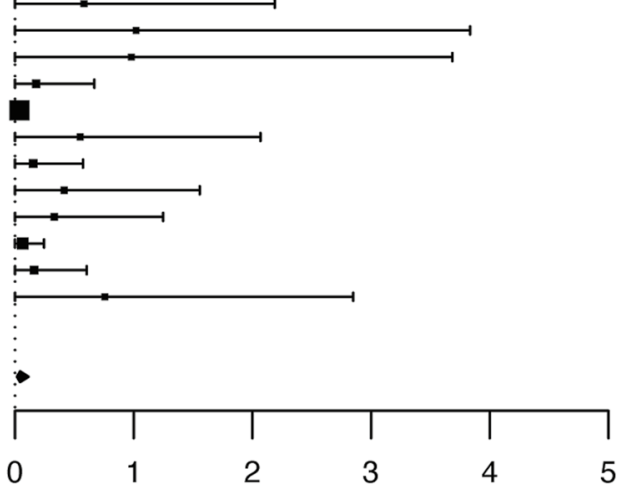

Pneumothorax incidence (\%)

$0.55[0.00,2.07$

$0.620 .00,2.32$

$0.180 .00,0.68$

$0.680 .00,2.54$

$0.860 .00,3.24$

$1.090 .00,4.08$

$0.720 .00,2.73$

0.21 0.00, 0.81

$0.220 .00,0.82$

$0.230 .00,0.87$

$0.200 .00,0.47$

$0.250 .00,0.92$

$0.100 .00,0.37$

$0.100 .00,0.29$

0.58 0.00, 2.19

$1.020 .00,3.83$

$0.980 .00,3.68$

$0.180 .00,0.67$

$0.040 .00,0.11$

$0.550 .00,2.07$

$0.150 .00,0.57$

$0.410 .00,1.56$

$0.330 .00,1.25$

$0.060 .00,0.24$

$0.160 .00,0.60$

$0.76[0.00,2.85$ ]

$0.04[0.02,0.11]$

B

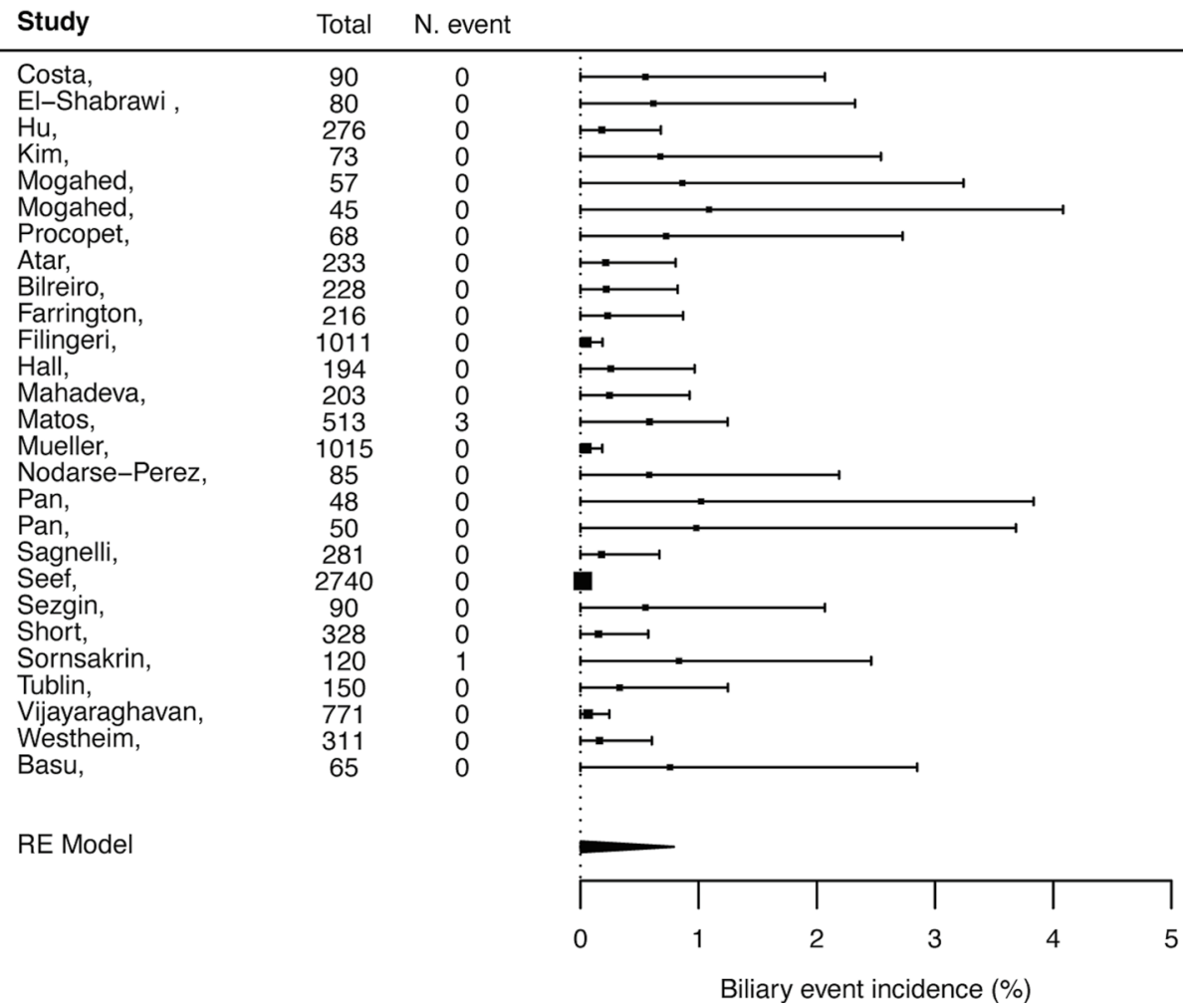

Estimate $[95 \% \mathrm{Cl}]$

$0.55[0.00,2.07]$

$0.62[0.00,2.32$

$0.18[0.00,0.68$

$0.68[0.00,2.54$

$0.86[0.00,3.24$

$1.09[0.00,4.08$

$0.72[0.00,2.73$

$0.21[0.00,0.81$

$0.22[0.00,0.82$

$0.23[0.00,0.87$

$0.05[0.00,0.19$

$0.26[0.00,0.97$

$0.25[0.00,0.92$

$0.58[0.00,1.24$

$0.05[0.00,0.19]$

$0.58[0.00,2.19$

$1.02[0.00,3.83$

$0.98[0.00,3.68$

$0.18[0.00,0.67$

$0.02[0.00,0.07$

$0.55[0.00,2.07$

0.15 [0.00, 0.57

$0.83[0.00,2.46$

$0.33[0.00,1.25$

$0.06[0.00,0.24$

$0.16[0.00,0.60$

$0.76[0.00,2.85$

$0.00[0.00,0.79]$

Fig. 7 Forest plots showing pooled incidence (as \%) for pneumothorax (a) and biliary events (b) 
A

Study

Total N. event

Estimate $[95 \% \mathrm{Cl}]$

Costa,

$\begin{array}{lr}\text { Costa, } & 90 \\ \text { El-Shabrawi }, & 80 \\ \mathrm{Hu}, & 276\end{array}$

90
80
276

Kim,

73

Mogahed

73

Mogahed

45

Procopet

68

Atar

233

Farrington,

216

Filingeri,

1011

Mahadeva,

203

Matos,

513

Mueller,

1015

Nodarse-Perez, $\quad 85$

Pan,

Pan,

Sagnelli,

48

50

Seef,

Sezgin,

281

2740

Short,

90

Sornsakrin $\quad 328$

Tublin, $\quad 150$

Vijayaraghavan, $\quad 771$

Basu,

771
65

RE Model

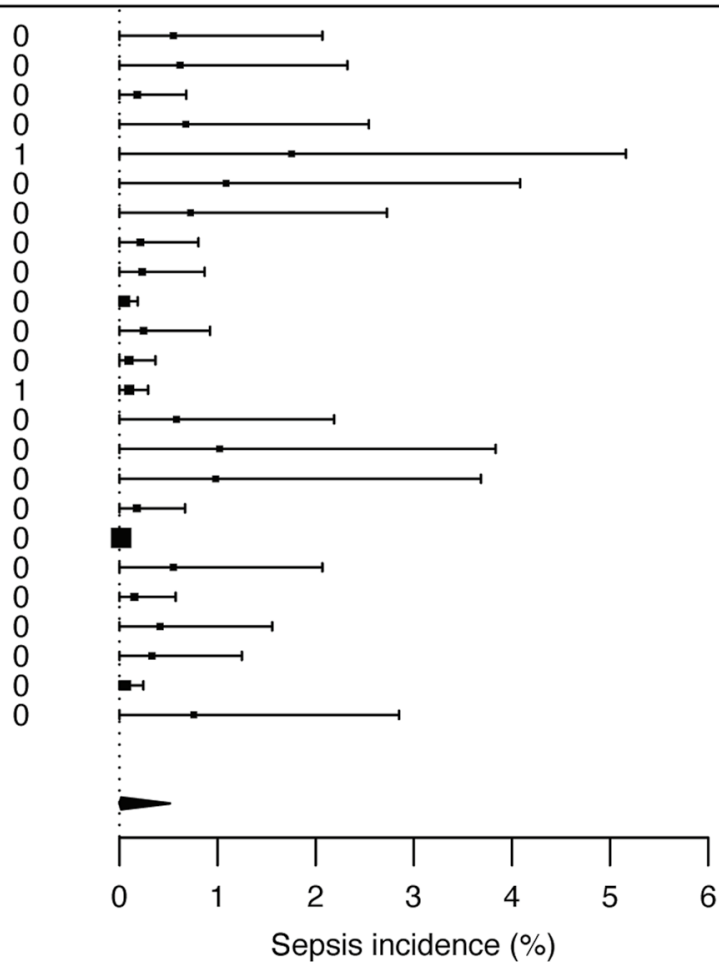

$0.55[0.00,2.07$

0.62 [0.00, 2.32]

$0.18[0.00,0.68$

$0.68[0.00,2.54$

$1.75[0.00,5.16$

$1.09[0.00,4.08$

$0.72[0.00,2.73]$

$0.21[0.00,0.81$

$0.23[0.00,0.87$

$0.05[0.00,0.19$

$0.25[0.00,0.92$

$0.10[0.00,0.37$

$0.10[0.00,0.29$

$0.58[0.00,2.19]$

$1.02[0.00,3.83$

$0.98[0.00,3.68$

$0.18[0.00,0.67]$

$0.02[0.00,0.07$

$0.55[0.00,2.07$

$0.15[0.00,0.57]$

$0.41[0.00,1.56$

$0.33[0.00,1.25$

$0.06[0.00,0.24]$

$0.76[0.00,2.85]$

$0.02[0.00,0.51]$

Sepsis incidence (\%)

B

Study

Total N. event

Estimate [95\% Cl]

Costa,

El-Shabrawi ,

$\mathrm{Hu}$,

Kim,

Mogahed,

Mogahed,

Procopet,

Atar,

Bilreiro,

Farrington,

Filingeri,

Hall,

Mahadeva,

Matos,

Mueller,

Nodarse-Perez,

$90 \quad 0$

$80 \quad 0$

276
73

57

45

45
68

233

228

216

1011

194

203

Pan,

Pan,

Sagnelli,

Seef,

Sezgin,

Sornsakrin,

Tublin,

Vijayaraghavan,

Westheim,

Basu,

85

48
50

281

90

120

150

771

311

65

RE Model

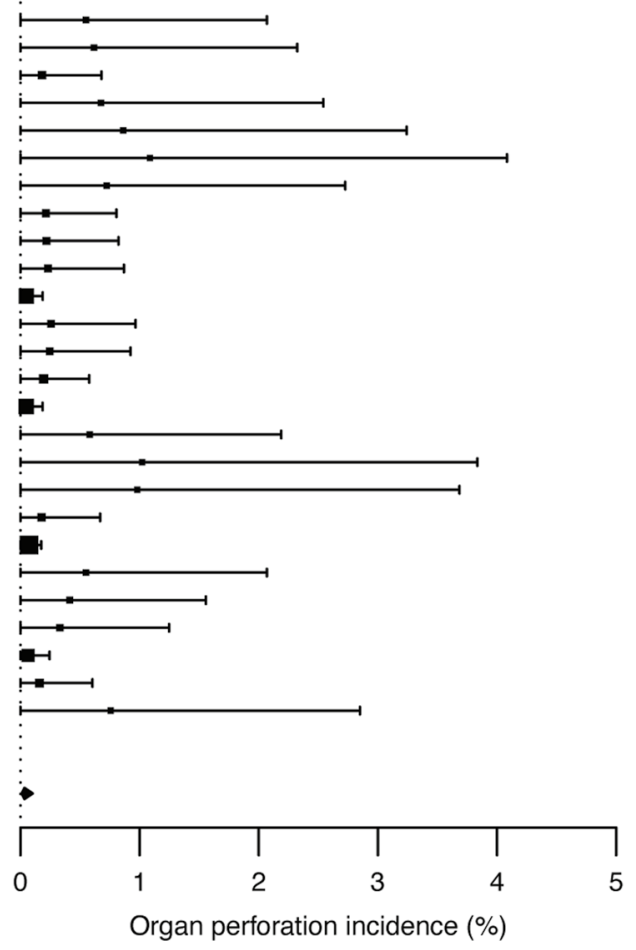

$0.55[0.00,2.07]$

$0.62[0.00,2.32$

$0.18[0.00,0.68]$

$0.68[0.00,2.54]$

$0.86[0.00,3.24$

$1.09[0.00,4.08]$

$0.72[0.00,2.73$

$0.21[0.00,0.81]$

$0.22[0.00,0.82$

$0.23[0.00,0.87$

$0.05[0.00,0.19$

$0.26[0.00,0.97$

$0.25[0.00,0.92]$

$0.19[0.00,0.58$

$0.05[0.00,0.19$

$0.58[0.00,2.19$

$1.02[0.00,3.83$

$0.98[0.00,3.68$

$0.18[0.00,0.67$

$0.07[0.00,0.17$

$0.55[0.00,2.07$

$0.41[0.00,1.56$

$0.33[0.00,1.25$

$0.06[0.00,0.24$

$0.16[0.00,0.60]$

$0.76[0.00,2.85]$

$0.03[0.01,0.10]$

Fig. 8 Forest plots showing pooled incidence (as \%) for sepsis (a) and organ perforation (b) 


\section{Minor Complications}

Minor complication incidence was 9.53\% (95\% CI 3.68, $22.5)$. Mild pain was the most frequent minor complication [12.9\% (95\% CI 5.34, 27.9), Table 2; Figs. 9, 10 and 11]. Potential publication bias was identified for all minor complications (Supplementary Fig. 3).

\section{Impact of Patient Age and Disease Severity on Complication Incidence}

Fitting mixed effects models based on study-level covariates reduced the heterogeneity across studies for most complications, with substantial or complete reduction for hospitalization, major bleeding, transfusion, major hematoma and technical failure (Table 3).

Decreasing population age significantly increased the incidence of major bleeding complications, with odds increasing fourfold in studies including pediatric patients or 16 -fold in pediatric only reports $(P$ values $<0.0001$; Tables 3 and 4). Similarly, for hospitalization, incidence was by twofold higher in studies including pediatric patients and 3.8-fold higher in pediatric only cohorts. This was corroborated by the frequency of all events reported in pediatric studies which was by far higher than in adult only studies (Table 2). Incidence of complications in the subset of pediatric only studies confirms this, with hospitalization incidence at $1.41 \%(0.57,3.47)$ and major bleeding at $2.15 \%(0.68$, 6.57) (Supplementary Table 4). In addition, hospitalization incidence was also impacted by the severity of the underlying disease, so that odds of hospitalization increased 1.25fold in studies with mainly post-transplant patients, 1.56-fold in patients with ascites and 1.95-fold in cirrhotic patients (Tables 3 and 4).

No significant impact was observed as a result of USguidance in the biopsy (biopsy type was below the 0.001 significance threshold in Tables 3 and 4), perhaps because many studies used US, even if only to assist ahead of the biopsy rather guide during biopsy. The year of publication impacted incidence reported for hospitalization, technical failure, mild pain and minor complications (Tables 3 and 4). Technical failure was impacted by the method of complication recording, so that odds of technical failure reduced by ninefold in studies were recording of complications was solicited rather than spontaneously reported by the patient (Tables 3 and 4).

\section{Other Confounders}

While we extracted data for additional confounders (BMI, higher risk of bleeding and sedation, operator experience), these parameters were insufficiently reported on $(4 / 30$,
$26 / 30,19 / 30$ and 16/30 studies, respectively) for full quantitative analysis (Supplementary Table 5). Nevertheless, a trend was seen such that hospitalization occurred more frequently in cohorts with higher bleeding risk and potential coagulopathy (77/9094 with healthy INR or platelets versus 26/1873 with poor coagulation profile). This same trend was also observed for major bleeding, with more frequent events in studies including patients with higher bleeding risk (79/9094 versus 19/1873). In contrast, hospitalization incidence appeared similar whether sedation was included or not (53/4955 with sedation versus 20/2064 with only local anesthetic), but major bleeding was more frequent with sedation (60/4955 versus 10/2064).

\section{Sensitivity Analysis}

Almost all studies were single-center studies thus potentially increasing the overall risk of bias, even though they tended to be smaller studies. In separate meta-analysis of complications from single-center studies (27/30 studies representing 8298 biopsies, Supplementary Table 6), most incidences remained very close to the original analysis that included multi-center studies. However, the incidences of major complications [3.04\% (95\% CI 0.97, 9.11)], technical failure [1.83\% (95\% CI 0.76, 4.33)], mild pain [16.6\% (95\% CI $6.97,34.7)]$, other minor events $[0.37 \%$ (95\% CI 0.10 , 1.42)] and all minor complications [13.1\% (95\% CI 4.82, 31.1)] were higher in the single-center analysis due to lack of relevant event reporting in the multi-center studies.

\section{Quality of Evidence}

Based on the GRADE approach [18], we found the quality of evidence for cohort age as a prognostic predictor of biopsy complications to be high and for disease severity to be moderate. The quality of evidence for death, hemoperitoneum, hemobilia, hemothorax, organ perforation, pneumothorax, sepsis and biliary complications were all judged to be low due to the sparse number of events or small number of reporting studies. Technical failure lacked consistency in sampling methods across studies. Major bleeding, hematoma, major complications and hospitalization were judged to have high quality evidence due to the frequency of reporting and the removal of potential bias following inclusion of cohort characteristics in the analysis. Sedation during biopsy was least reported and was considered the main source of potential bias. 
A

Study

Total N. event

Estimate $[95 \% \mathrm{Cl}]$

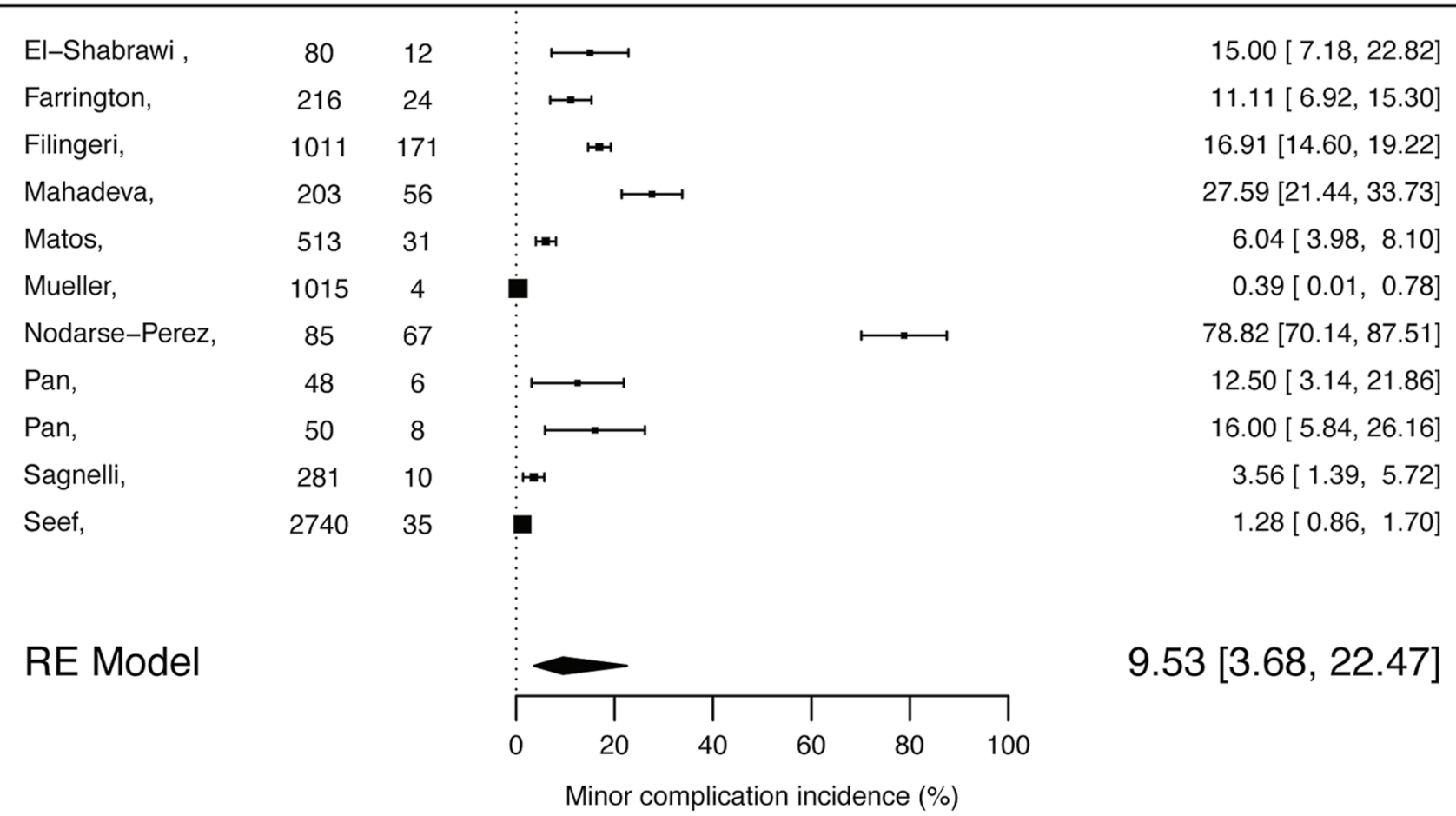

B

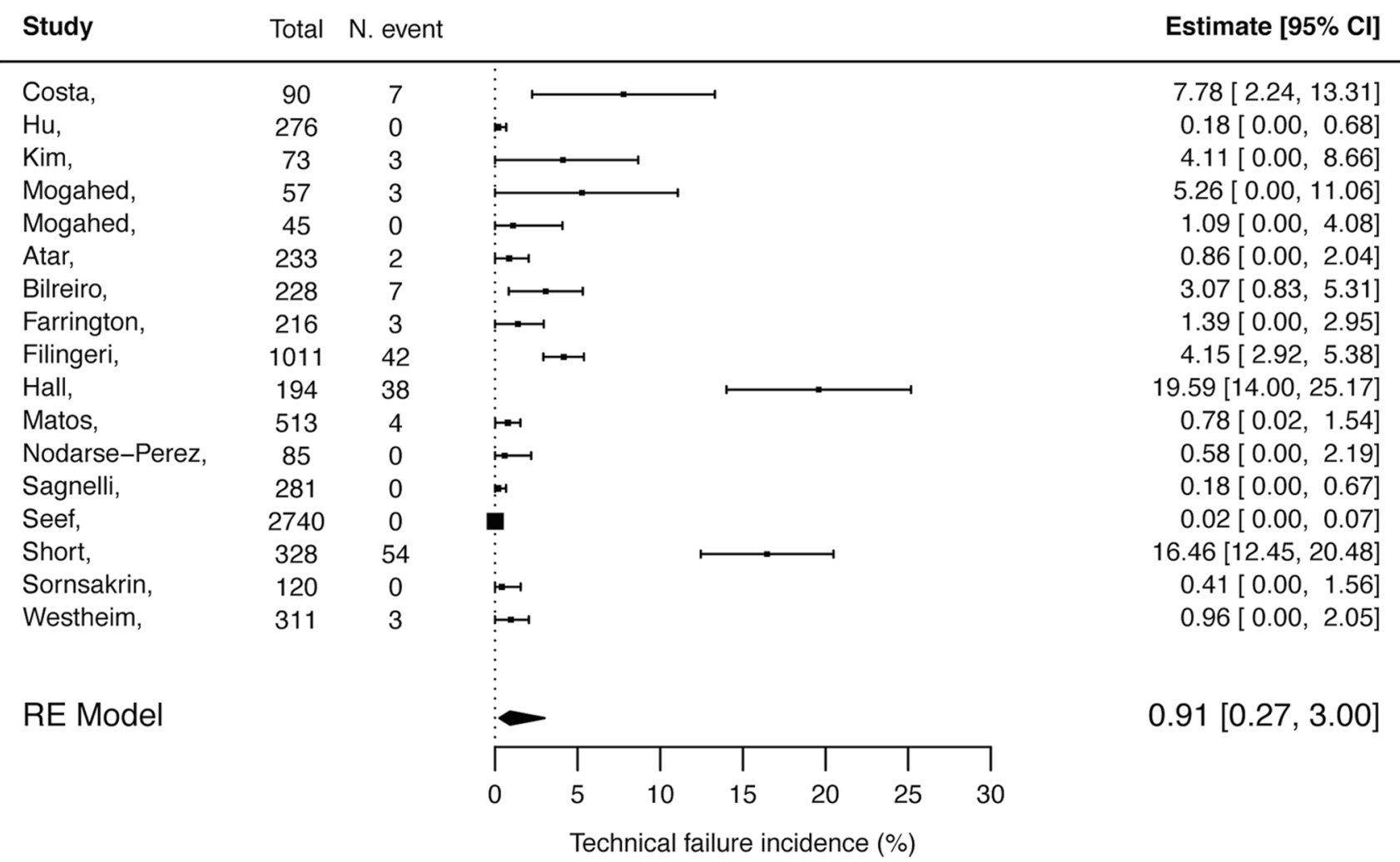

Fig. 9 Forest plots showing pooled incidence (as \%) for all minor complications (a) and technical failure (b) 
A

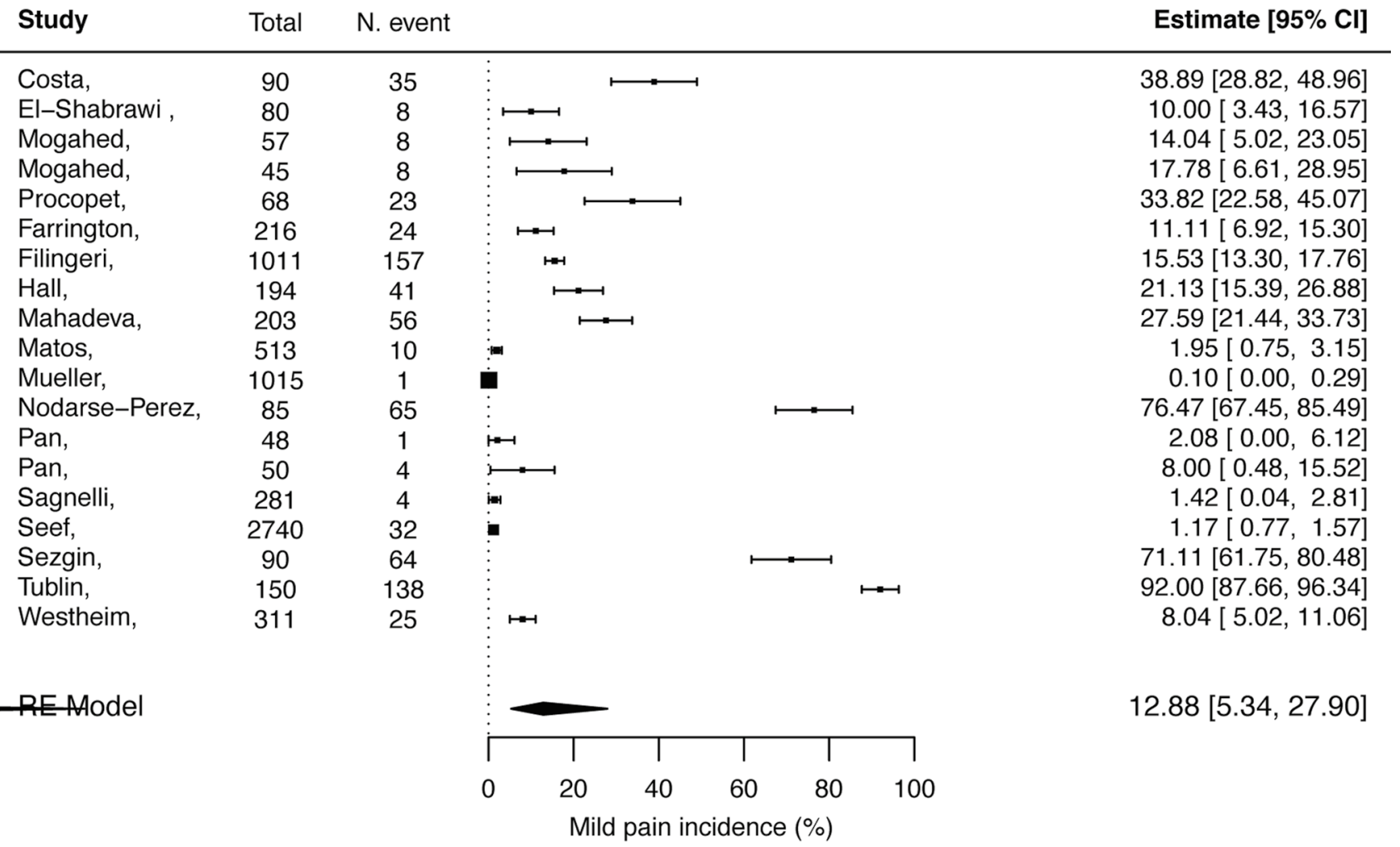

B

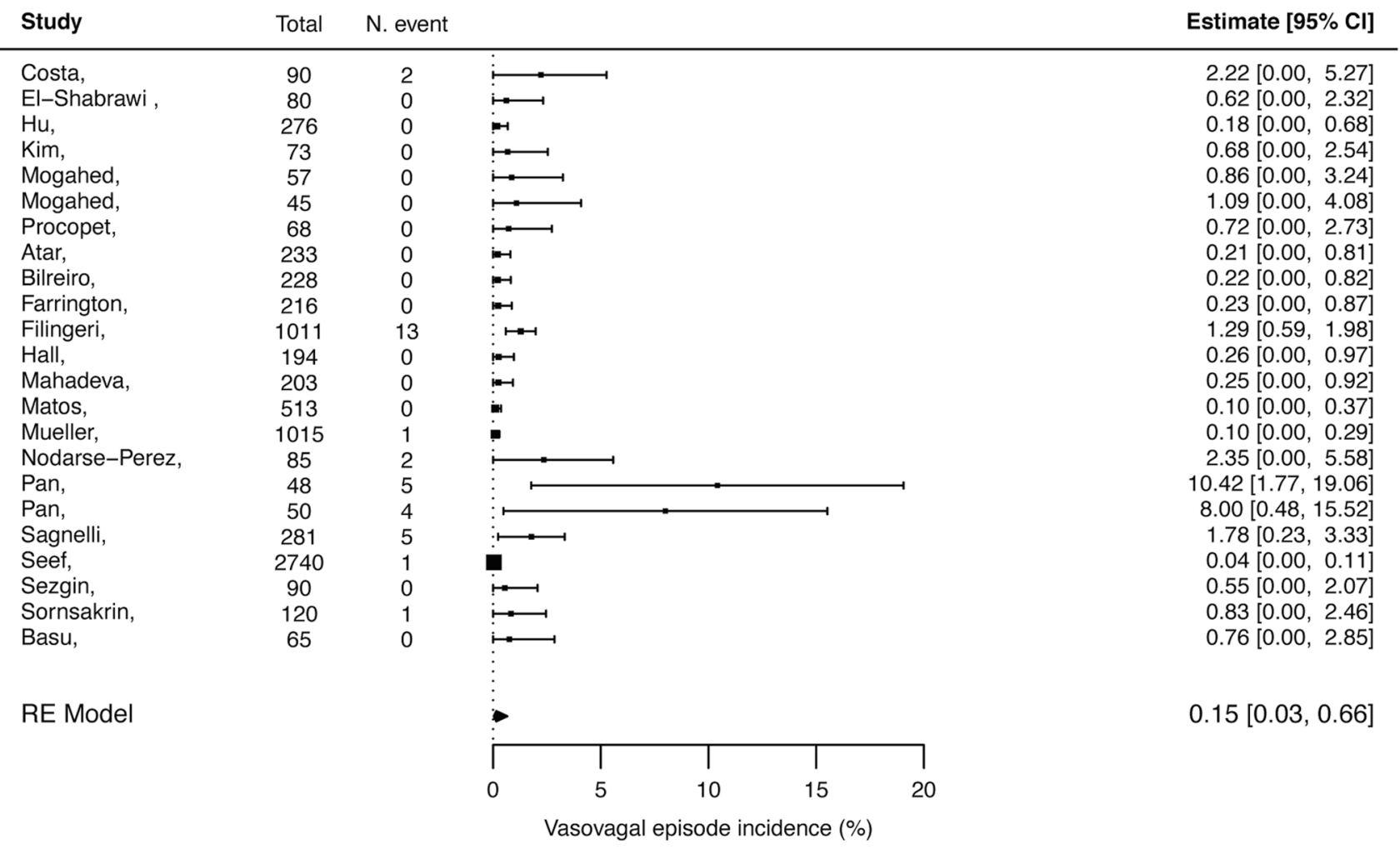

Fig. 10 Forest plot showing pooled incidence (as \%) for mild pain (a) and vasovagal episodes (b) 
A

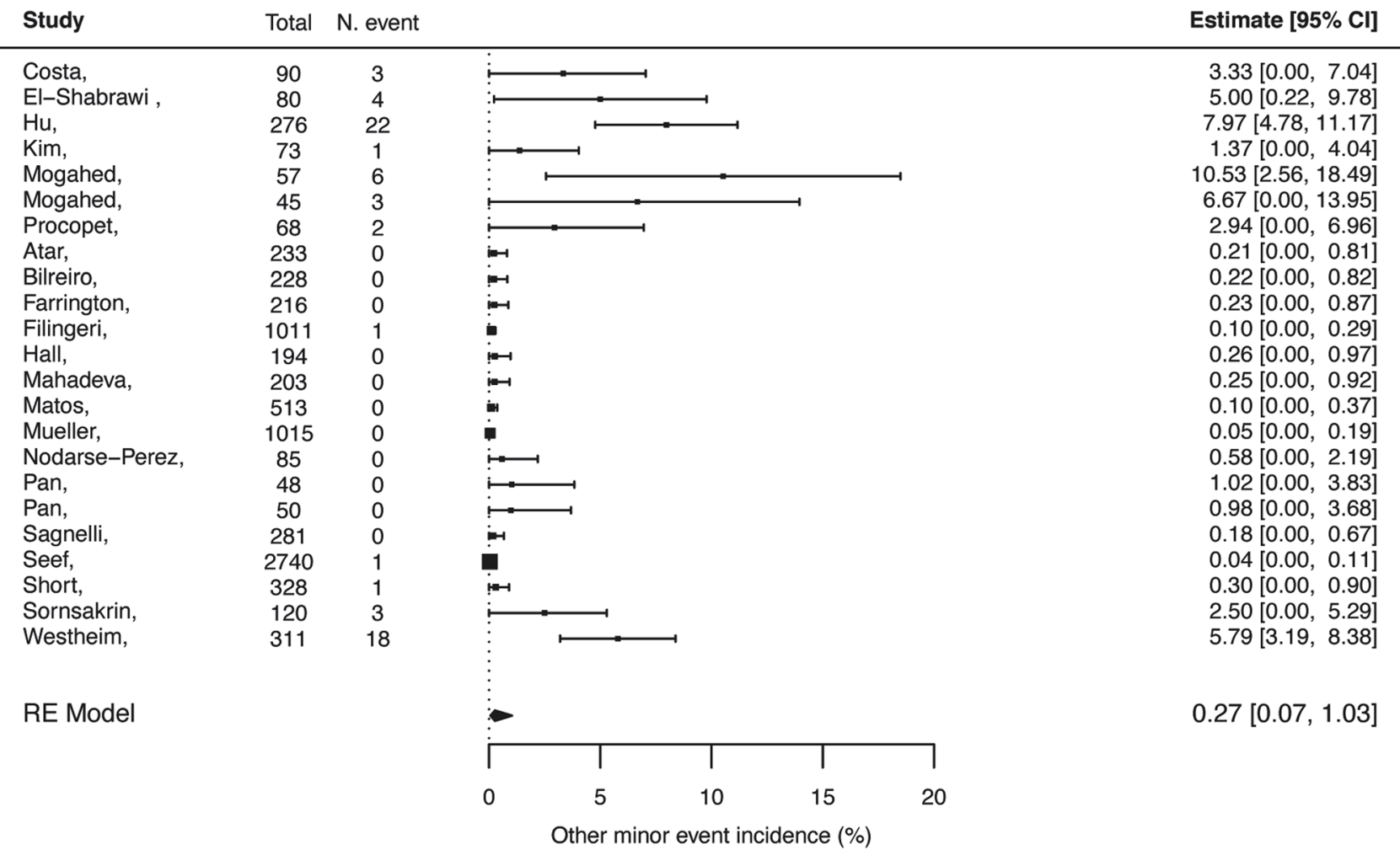

B

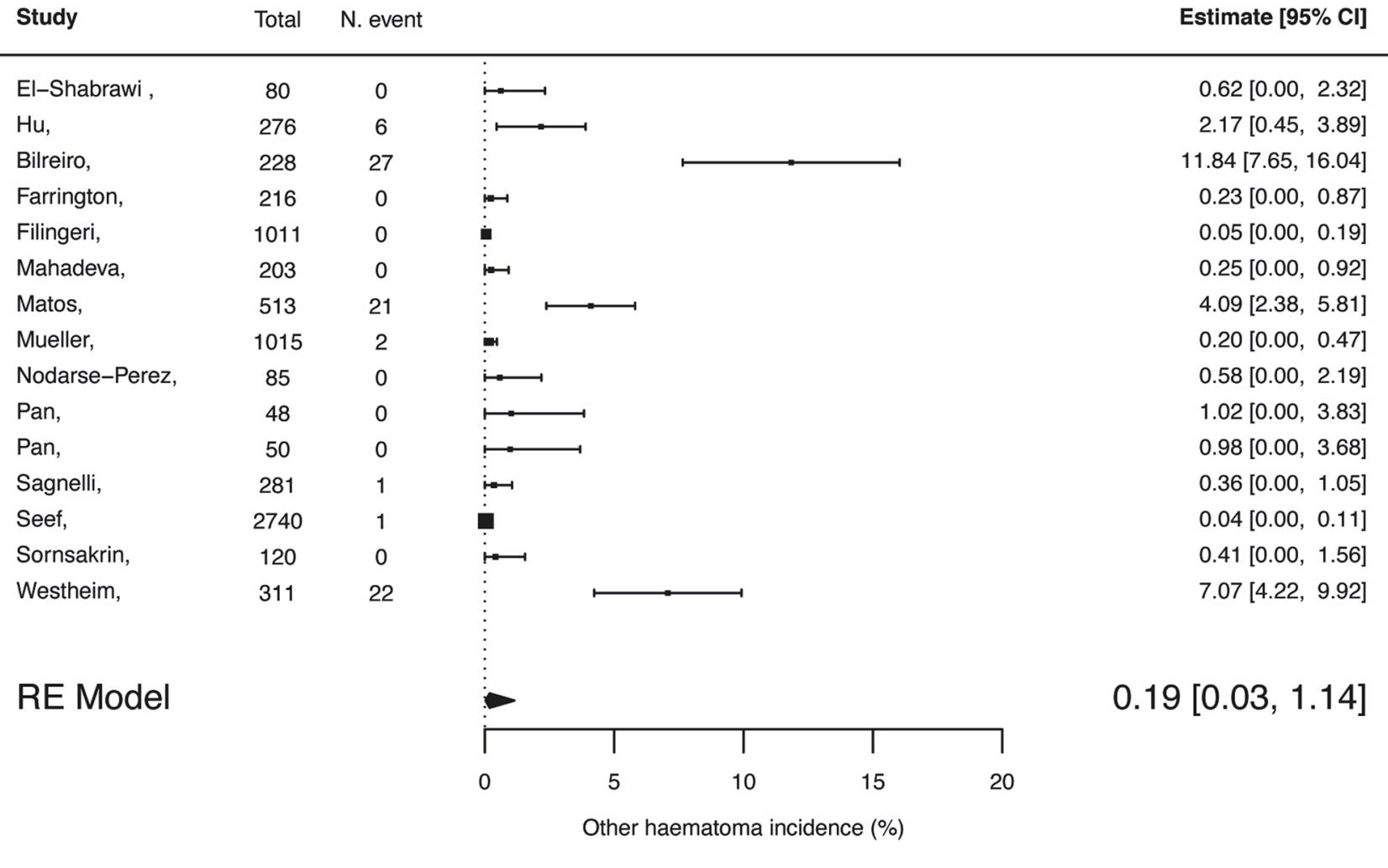

Fig. 11 Forest plot showing pooled incidence (as \%) for other minor complications (a) and other hematoma (b) 
Table 3 Impact of publication year, population age, disease severity, outcome collection (solicited or spontaneous) and biopsy type (percutaneous with or without US-guidance) on incidence of complications

\begin{tabular}{|c|c|c|c|c|c|c|c|c|c|}
\hline \multirow[t]{2}{*}{ Event } & \multicolumn{7}{|l|}{$P$ value $^{\mathrm{a}}$} & \multirow{2}{*}{$\begin{array}{l}\text { Origi- } \\
\text { nal } I^{2} \\
(\%)\end{array}$} & \multirow{2}{*}{$\begin{array}{l}\text { Residual } \\
I^{2}(\%)^{\mathrm{b}}\end{array}$} \\
\hline & $\begin{array}{l}\text { QM-test } \\
\text { of covari- } \\
\text { ates }\end{array}$ & $\begin{array}{l}\text { Publication } \\
\text { year }\end{array}$ & $\begin{array}{l}\text { Population } \\
\text { age }\end{array}$ & $\begin{array}{l}\text { Disease sever- } \\
\text { ity }\end{array}$ & $\begin{array}{l}\text { Outcome col- } \\
\text { lection }\end{array}$ & Biopsy type & $\begin{array}{l}\text { Residual } \\
\text { heterogeneity } \\
\text { (LRT-test) }\end{array}$ & & \\
\hline $\begin{array}{l}\text { Hospitaliza- } \\
\text { tion }\end{array}$ & $<.0001$ & $<.0001$ & $<0.0001$ & 0.0007 & 0.0033 & 0.0833 & 0.0004 & 86 & 0 \\
\hline $\begin{array}{l}\text { Moderate/ } \\
\text { severe pain }\end{array}$ & 0.1555 & 0.1154 & 0.2451 & 0.3362 & 0.5547 & 0.0311 & $<.0001$ & 96 & 87 \\
\hline $\begin{array}{l}\text { Major bleed- } \\
\text { ing }\end{array}$ & $<.0001$ & 0.4799 & $<.0001$ & 0.5294 & 0.1175 & 0.0892 & $<.0001$ & 94 & 57 \\
\hline Transfusion & 0.0306 & 0.7782 & 0.0016 & 0.9402 & 0.9512 & 0.1267 & 0.0561 & 80 & 41 \\
\hline $\begin{array}{l}\text { Major } \\
\text { hematoma }^{c}\end{array}$ & 0.0780 & 0.2823 & 0.0191 & 0.7475 & 0.7131 & 0.2789 & $<.0001$ & 90 & 70 \\
\hline $\begin{array}{l}\text { Other } \\
\text { hematoma }^{\mathrm{d}}\end{array}$ & 0.0177 & 0.7460 & 0.0586 & 0.8184 & 0.2373 & 0.6478 & $<.0001$ & 97 & 85 \\
\hline $\begin{array}{l}\text { All major } \\
\text { events }\end{array}$ & 0.2273 & 0.1469 & 0.2198 & 0.3229 & 0.4015 & 0.8049 & $<.0001$ & 97 & 88 \\
\hline $\begin{array}{l}\text { Technical } \\
\text { failure }\end{array}$ & $<.0001$ & $<.0001$ & 0.0015 & 0.0230 & $<.0001$ & 0.1278 & $<.0001$ & 97 & 58 \\
\hline Mild pain & 0.0002 & $<.0001$ & 0.7771 & 0.6829 & 0.7900 & 0.1901 & $<.0001$ & 99 & 96 \\
\hline $\begin{array}{r}\text { Vasovagal } \\
\text { episodes }\end{array}$ & 0.1625 & 0.3345 & 0.1185 & 0.6103 & 0.9617 & 0.2523 & $<.0001$ & 89 & 75 \\
\hline $\begin{array}{l}\text { Other minor } \\
\text { events }^{\mathrm{e}}\end{array}$ & 0.0027 & 0.9610 & 0.0160 & 0.9305 & 0.0382 & 0.0207 & $<.0001$ & 94 & 77 \\
\hline All minor & $<.0001$ & 0.0002 & 0.0769 & 0.8692 & 0.1990 & 0.0613 & $<.0001$ & 99 & 90 \\
\hline
\end{tabular}

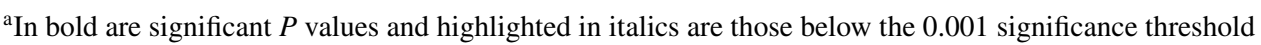

${ }^{\mathrm{b}}$ After covariate adjustment

${ }^{\mathrm{c}}$ Subcapsular, intramuscular, intrahepatic, variceal hematoma

${ }^{\mathrm{d}}$ Hematoma at biopsy site, transthoracic, cervical hematoma

${ }^{\mathrm{e}}$ Arteriovenous fistula, fever, other (not pain or vasovagal episodes)

Table 4 Estimates of odds with 95\% CI showing potential contribution of individual covariates on the incidence on complications

\begin{tabular}{|c|c|c|c|c|c|}
\hline \multirow[t]{2}{*}{ Event } & \multicolumn{5}{|c|}{ Odds estimate $(95 \% \mathrm{CI})$ by covariate ${ }^{\mathrm{a}}$} \\
\hline & Publication year & Population age & Disease severity & Outcome collection & Biopsy type \\
\hline Hospitalization & $1.15(1.08,1.22)$ & $2.22(1.62,3.04)$ & $1.25(1.10,1.43)$ & $0.47(0.28,0.78)$ & $0.62(0.36,1.07)$ \\
\hline Moderate/severe pain & $1.47(0.92,2.35)$ & $0.42(0.10,1.83)$ & $2.01(0.49,8.24)$ & $0.51(0.06,4.80)$ & $11.5(1.25,105)$ \\
\hline Major bleeding & $1.07(0.91,1.25)$ & $4.00(2.36,6.77)$ & $0.85(0.51,1.43)$ & $0.48(0.19,1.21)$ & $0.48(0.21,1.13)$ \\
\hline Transfusion & $0.96(0.68,1.35)$ & $5.35(1.89,15.2)$ & $0.96(0.33,2.84)$ & $0.94(0.12,7.63)$ & $0.22(0.04,1.55)$ \\
\hline Major hematoma $^{\mathrm{b}}$ & $1.22(0.86,1.73)$ & $3.52(1.23,10.0)$ & $1.23(0.36,4.30)$ & $1.63(0.13,21.4)$ & $0.36(0.06,2.33)$ \\
\hline Other hematoma ${ }^{\mathrm{c}}$ & $1.08(0.69,1.71)$ & $3.59(0.96,13.4)$ & $1.21(0.25,5.80)$ & $10.3(0.22,490)$ & $0.52(0.04,8.75)$ \\
\hline All major events & $1.31(0.92,1.88)$ & $2.15(0.64,7.23)$ & $1.93(0.53,7.02)$ & $3.71(0.18,78.9)$ & $0.75(0.08,7.78)$ \\
\hline Technical failure & $1.77(1.43,2.18)$ & $2.82(1.49,5.32)$ & $0.38(0.16,0.88)$ & $0.07(0.03,0.19)$ & $2.37(0.79,7.19)$ \\
\hline Mild pain & $1.78(1.34,2.37)$ & $0.89(0.39,2.04)$ & $1.22(0.48,3.12)$ & $1.29(0.21,8.10)$ & $2.51(0.64,9.86)$ \\
\hline Vasovagal episodes & $1.21(0.83,1.76)$ & $0.29(0.06,1.39)$ & $0.70(0.18,2.79)$ & $1.07(0.09,13.6)$ & $3.61(0.41,32.3)$ \\
\hline Other minor events ${ }^{\mathrm{d}}$ & $1.00(0.74,1.35)$ & $3.47(1.27,9.51)$ & $0.94(0.21,4.30)$ & $14.5(1.16,180)$ & $0.08(0.01,0.67)$ \\
\hline All minor & $1.97(1.39,2.80)$ & $2.2(0.92,5.23)$ & $0.95(0.46,1.93)$ & $0.37(0.08,1.72)$ & $3.43(0.95,12.5)$ \\
\hline
\end{tabular}

${ }^{a}$ In bold are those with significant $P$ values and highlighted in italics are those below the 0.001 significance threshold

${ }^{\mathrm{b}}$ Subcapsular, intramuscular, intrahepatic, variceal hematoma

${ }^{\mathrm{c}}$ Hematoma at biopsy site, transthoracic, cervical hematoma

${ }^{\mathrm{d}}$ Arteriovenous fistula, fever, other (not pain or vasovagal episodes) 


\section{Discussion}

This systematic review and meta-analysis describe accurate, contemporary incidences for all complications reported to result from percutaneous liver biopsy, in 16 individual clinical categories. Additionally, incidences of hospitalization and technical failure have for the first time been provided following meta-analysis rather than based on local, singlecenter reports. These incidences provide current standardized figures for assessment of the impact that continued inclusion of liver biopsy in clinical care has on diagnosis, staging and monitoring of chronic liver disease. At 24 major complications per 1000 patients and 90 minor complications per 1000 patients, these current incidences on the most commonly performed biopsy procedure are substantial.

Our updated analysis has confirmed that death incidence is $0.01 \%$. This represents 12 reported deaths in 64,356 biopsy procedures, and concurs with original reports from 1986 [10]. Additionally, hemoperitoneum, hemobilia, hemothorax, organ perforation, pneumothorax, sepsis and biliary complications continue to be rare $[3,12]$. The updated incidence of major bleeding at $0.48 \%$ (95\% CI $0.22,1.06$ ) is in line with a 2019 scoping review for US-guided percutaneous biopsies quoting a range between $0.1 \%$ and $4.6 \%$ [3]. Incidence of major hematoma reported in our work is substantial $[0.11 \%(95 \%$ CI $0.02,0.53)]$, as is that of minor hematoma $[0.19 \%$ (95\% CI 0.03, 1.14)]. Reporting of minor hematoma was absent in many of the articles in our review (reported in 17/39 studies) and ultrasound follow-up of biopsied patients was not standard procedure in most studies. Thus, the incidence of hematoma is likely to be higher than presented here and changes to practice to standardize follow-up are advocated.

Both major $(0.48 \%)$ and minor $(0.19 \%)$ bleeding in our study occurred more frequently than either major bleeding $(0.00 \%)$ or minor bleeding $(0.00 \%)$ in a meta-analysis of US-assisted percutaneous biopsies with a wider study period (1978-2017) [12]. That work included studies of focal disease patients (24/51 cited articles), where sampling occurs during surgery and is targeted to the tumor area. Just over half of the included articles in that meta-analysis were published before 2010 (26/51) and studies without US were for the main absent, thus limiting overlap with this work. We found no evidence in our analysis that US assistance significantly reduced complication rates, precluding that as the explanation of differences in incidence. Instead, there was a fundamental limitation in the earlier work [12] which was that no adjustment of sample size or study selection was made to account for gaps in reporting, thus giving rise to negligible complication incidences. In contrast, in the present work major bleeding included specific, clinically defined bleeding events and study selection was adjusted by complication category. This was to reduce subjectivity resulting from differences in local hospital guidelines and reporting, as our qualitative review revealed that classification of major, mild and "non-significant" events varied widely between studies, and details of clinical follow-up for complications were infrequently reported.

We also report higher values for pain $(0.34 \%$ for moderate/severe pain and for $12.9 \%$ mild pain) compared to the $0.00 \%$ incidence in that earlier meta-analysis [12] for the reasons stated above regarding scope and methodology. While frequent, pain was reported in only 21 of 30 studies included in our work and its incidence varied most between studies. Additionally, sedation and/or analgesia was not sufficiently reported for quantitative assessment of its impact on patient pain. Several studies introduced the VAS scale and ensured consideration of this complication by solicited questionnaires. Pain is difficult to assess objectively and is potentially affected by recall bias and the frequency of follow-up [30]; nevertheless, to the patient this complication is a key consideration and would affect the interaction with the clinician. Improvements in clinical recording of pain and prospective studies investigating in detail the quality, nature and duration of pain are required to enable fully informed patient consent.

Technical failure at $0.91 \%$ can indirectly affect biopsy complications, as well as patient diagnosis and disease monitoring [31]. Here we found that studies in which reporting of complications from the patient were solicited by the clinical team, had lower odds of technical failures, and we speculate that this was indicative of exceptional care for patient wellbeing. In our review, sample adequacy was inconsistently reported across studies (5-10 portal tracks, $12-18 \mathrm{~mm}$, number of fragments) and needle passes varied (2-6) or were infrequently reported so firm conclusions as to causes cannot be drawn. Nevertheless, the incidence reported here is much lower than that reported in a meta-analysis of endoscopic US-guided biopsies (10\% with inadequate specimen) [32].

Until this work, there was weak evidence to support that age, coagulation status and comorbidities impact the incidence of percutaneous liver biopsy complications [3]. On fuller analysis we find that incidence of hospitalization (defined by additional, non-routine care including severe adverse events) and major bleeding increased most dramatically in pediatric patients. The same conclusion was drawn when the few transjugular and endoscopic biopsy studies identified during this systematic review were included (Thomaides-Brears unpublished). In addition, increased odds of hospitalization were observed in patients with more advanced disease. Thus, patients with cirrhosis are 4 times more likely to be hospitalized after biopsy, compared to patients with earlier stage disease such as NAFLD. While not investigated in this work, complications also arise in non-liver disease indications where liver biopsies are the 
diagnostic method, with transfusions reported in 25-30\% during pre-recovery or pre-surgery biopsy in liver transplant donors [33]. In percutaneous liver biopsy to pre-empt liver damage in pediatric hematopoietic stem cell transplant patients, $17 \%$ exhibited mild pain and/or minor hematoma [34]. These findings advocate avoidance of liver biopsy as a recurring event, although incidences reported here are lower than for renal biopsies, in which hematomas occur in $11 \%$ and mild pain in $4.3 \%$ of patients [35]. While others have found that sedation increased bleeding due to resulting hypoxia and lower hematocrit [36], there was insufficient reporting for us to draw significant conclusions, though there was a trend towards this.

The limitations of this study include the residual heterogeneity in incidences between studies that reduce the quality of the evidence for some events, particularly for rare events such as death and transfusion, and to which reporting bias is a key contributor. Unaccounted risk factors that might explain these limitations are differences in needle type and size, and technical expertise for all procedures, which were too variable to accurately include in this meta-analysis. Other differences between procedures such as plugged or blind biopsies could also account for the heterogeneity. Nevertheless, inclusion of cohort age, disease severity, outcome collection details and publication year as covariates in our analyses removed heterogeneity completely for the incidence of hospitalization and reduced the level of heterogeneity for major bleeding and hematoma. Future applications of this work could include the derivation of a risk score to predict the probability of biopsy complications that takes account of cohort characteristics as prognostic factors. Addition of BMI, a risk factor for fatty liver disease outcomes [37] would be a very relevant risk factor.

In conclusion, this study provides current incidences for complications resulting from percutaneous liver biopsy procedures performed for patients with chronic liver disease; $2.4 \%$ for major and $9.5 \%$ for minor complications. We believe this data will inform future decisions in clinical care for such patients and highlight the need for more clearly defined formal indications with both non-invasive techniques and liver biopsies as part of diagnostic algorithms. These data should be included in future health economic analyses aiming to inform clinical guidelines and reduce adverse patient outcomes.

Supplementary Information The online version contains supplementary material available at https://doi.org/10.1007/s10620-021-07089-w.

Acknowledgments The authors wish to gratefully acknowledge the contributions of David DeBrota, Tom Nichols, Trit Garg, Sam Nussbaum, Laura Herdman, Lewis Jenkins, Gemma Greenall, Amna Soomro, Ma'Leah Dunn, Maria Salazar, Louise Tetlow, Matt Kelly and Rajarshi Banerjee in critical review and preparation of the final manuscript.

\section{Declarations}

Conflict of interest No funding was received for this work. HTB, MF, $\mathrm{CP}$ and SF are employees of Perspectum Ltd, a privately funded commercial enterprise that develops medical devices to address unmet clinical needs, including LiverMultiScan ${ }^{\circledR}$. DRHC is an employee of the Global Liver Institute, a non-profit organization. $\mathrm{MN}$ has been on the advisory board for Gilead, Intercept, Pfizer, Fractyl, Novartis, Allergan, Blade, Echosens, EchoSens North America, OWL, Siemens, Roche diagnostic and Abbott; Speaker: Abbott, Echosens, Intercept. MN has received research support from Allergan, BMS, Gilead, Galmed, Galectin, Genfit, Conatus, Enanta, Novartis, Shire and Zydus; MN is a minor shareholder or has stocks in Anaetos and Viking. All other authors have nothing to disclose.

Open Access This article is licensed under a Creative Commons Attribution-NonCommercial 4.0 International License, which permits any non-commercial use, sharing, adaptation, distribution and reproduction in any medium or format, as long as you give appropriate credit to the original author(s) and the source, provide a link to the Creative Commons licence, and indicate if changes were made. The images or other third party material in this article are included in the article's Creative Commons licence, unless indicated otherwise in a credit line to the material. If material is not included in the article's Creative Commons licence and your intended use is not permitted by statutory regulation or exceeds the permitted use, you will need to obtain permission directly from the copyright holder. To view a copy of this licence, visit http://creativecommons.org/licenses/by-nc/4.0/.

\section{References}

1. Rockey DC, Caldwell SH, Goodman ZD, Nelson RC, Smith AD. Liver biopsy. Hepatology 2009;49:1017-1044.

2. Neuberger J, Patel J, Caldwell H, Davies S, Hebditch V, Hollywood $\mathrm{C}$ et al. Guidelines on the use of liver Guidelines on the use of liver biopsy in clinical practice from the British Society of Gastroenterology, the Royal College of Radiologists and the Royal College of Pathology. Gut 2020;69:1382 LP - 1403.

3. Midia M, Odedra D, Shuster A, Midia R, Muir J. Predictors of bleeding complications following percutaneous imageguided liver biopsy: a scoping review. Diagn Interv Radiol 2019;25:71-80.

4. Dezsőfi A, Baumann U, Dhawan A, Durmaz O, Fischler B, Hadzic $\mathrm{N}$ et al. Liver biopsy in children: position paper of the ESPGHAN Hepatology Committee. J Pediatr Gastroenterol Nutr 2015;60:408-420.

5. Pavlides M, Birks J, Fryer E, Delaney D, Sarania N, Banerjee R et al. Interobserver variability in histologic evaluation of liver fibrosis using categorical and quantitative scores. Am J Clin Pathol 2017;147:364-369.

6. Harrison SA, Wong VW-S, Okanoue T, Bzowej N, Vuppalanchi $\mathrm{R}$, Younes $\mathrm{Z}$ et al. Selonsertib for patients with bridging fibrosis or compensated cirrhosis due to NASH: results from randomized phase III STELLAR trials. J Hepatol 2020;73:26-39.

7. Goodman Z. Grading and staging systems for inflammation and fibrosis in chronic liver diseases. J Hepatol 2007;47:598-607.

8. Kleiner D, Brunt E, Van Natta M, Behling C, Contos M, Cummings $\mathrm{O}$ et al. Design and validation of a histological scoring system for nonalcoholic fatty liver disease. Hepatology 2005;41:1313-1321.

9. Standish RA, Cholongitas E, Dhillon A, Burroughs AK, Dhillon AP. An appraisal of the histopathological assessment of liver fibrosis. Gut. 2006;55:569-578. 
10. Piccinino F, Sagnelli E, Pasquale G, Giusti G, Battocchia A, Bernardi $\mathrm{M}$ et al. Complications following percutaneous liver biopsy: a multicentre retrospective study on 68276 biopsies. J Hepatol 1986;2:165-173.

11. Eisenberg E, Konopniki M, Veitsman E, Kramskay R, Gaitini D, Baruch Y. Prevalence and characteristics of pain induced by percutaneous liver biopsy. Anesth Analg 2003;96:1392-1396.

12. Tian G, Kong D, Jiang T, Li L. Complications after percutaneous ultrasound-guided liver biopsy. J Ultrasound Med. 2020. https:// doi.org/10.1002/jum.15229.

13. Moher D, Liberati A, Tetzlaff J, Altman DG. Preferred reporting items for systematic reviews and meta-analyses: The PRISMA statement. Ann Intern Med 2009;151:264-269.

14. Zakeri N, Tsochatzis EA. Bleeding risk with invasive procedures in patients with cirrhosis and coagulopathy. Curr Gastroenterol Rep 2017;19:45.

15. Hayden JA, Côté P, Bombardier C. Evaluation of the quality of prognosis studies in systematic reviews. Ann Intern Med 2006; 144:427-437.

16. Wells G, Shea B, O'Connell D, Peterson J, Welch V, Losos $\mathrm{M}$, et al. The Newcastle-Ottawa Scale (NOS) for assessing the quality of nonrandomised studies in meta-analyses [Internet]. 2013 [cited 2020 Jun 1]. http://www.ohri.ca/programs/clinical_ epidemiology/oxford.asp.

17. Cochrane. Cochrane Handbook for Systematic Reviews of Interventions version 6.0 (updated August 2019). 2019. www.train ing.cochrane.org/handbook. Last accessed 5th June 2020.

18. Iorio A, Spencer FA, Falavigna M, Alba C, Lang E, Burnand $B$ et al. Use of GRADE for assessment of evidence about prognosis: rating confidence in estimates of event rates in broad categories of patients. BMJ Br Med J 2015;350:h4870.

19. Hunter JP, Saratzis A, Sutton AJ, Boucher RH, Sayers RD, Bown MJ. In meta-analyses of proportion studies, funnel plots were found to be an inaccurate method of assessing publication bias. J Clin Epidemiol 2014;67:897-903.

20. Viechtbauer W, López-López JA, Sánchez-Meca J, MarínMartínez F. A comparison of procedures to test for moderators in mixed-effects meta-regression models. Psychol Methods 2015;20:360-374

21. Basu P, Nair T, Krishnaswamy N, Shah N, Brown R. Single use of romiplostim thrombopoietin analogue(TPO) in severe thrombocytopenia for outpatient percutaneous liver biopsy in patients with chronic liver disease (CLD)-a randomized double blinded prospective clinical pilot trial. Surg Endosc Other Interv Tech 2012;26:S333

22. Tublin ME, Blair R, Martin J, Malik S, Ruppert K, Demetris A. Prospective study of the impact of liver biopsy core size on specimen adequacy and procedural complications. Am J Roentgenol 2017;210:183-188.

23. Sezgin O, Yaras S, Ates F, Altintas E, Saritas B. Effectiveness of sedoanalgesia in percutaneous liver biopsy premedication. Eur J Hepato Gastroenterol 2017;7:146-149.

24. Sagnelli E, Sagnelli C, Pisaturo MA, Coppola N, Pasquale G, Piccinino $\mathrm{F}$ et al. Liver biopsy in chronic hepatitis $\mathrm{C}$ : the experience of 15 Italian wards of infectious diseases. Le Infez Med 2012;20:31-36

25. Mueller M, Kratzer W, Oeztuerk S, Wilhelm M, Mason RA, Mao R et al. Percutaneous ultrasonographically guided liver punctures: an analysis of 1961 patients over a period of ten years. BMC Gastroenterol 2012;12:173.

26. Mahadeva S, Mahfudz AS, Vijayananthan A. Ethnicity influences pain after ultrasound-guided percutaneous liver biopsy. Eur J Gastroenterol Hepatol. 2015;27:1378-1381.

27. Farrington EA, Maskell G, Hussaini HS. Feasibility and experience of nurse-led ultrasound-guided percutaneous liver biopsy. Frontline Gastroenterol 2012;3:187-190.
28. El-Shabrawi MH, El-Karaksy HM, Okahsa SH, Kamal NM, El-Batran G, Badr KA. Outpatient blind percutaneous liver biopsy in infants and children: is it safe? Saudi J Gastroenterol 2012;18:26-33.

29. Costa RS, Cardoso AF, Ferreira A, Costa J, Costa D, Fernandes $\mathrm{D}$, et al. What recovery position should patients adopt after percutaneous liver biopsy? Eur J Gastroenterol Hepatol 2019;31(2):253-259

30. Akay S, Karasu Z, Noyan A, Pala S, Musoglu A, İler T et al. Liver biopsy: Is the pain for real or is it only the fear of it? Dig Dis Sci 2007;52:579-581. https://doi.org/10.1007/ s10620-006-9493-6

31. Davison BA, Harrison SA, Cotter G, Alkhouri N, Sanyal A, Edwards C et al. Suboptimal reliability of liver biopsy evaluation has implications for randomized clinical trials. J Hepatol. 2020. https://doi.org/10.1016/j.jhep.2020.06.025.

32. Mohan BP, Shakhatreh M, Garg R, Ponnada S, Adler DG. Efficacy and safety of EUS-guided liver biopsy: A systematic review and meta-analysis. Gastrointest Endosc 2019;89:238-246.e3.

33. Oliver JB, Machineni P, Bongu A, Patel T, Nespral J, Kadric C et al. Liver biopsy in assessment of extended criteria donors. Liver Transplant 2018;24:182-191.

34. Maximova N, Gregori M, Barbieri F, Pizzol A, Sonzogni A. Safety and utility of percutaneous liver biopsy in hematopoietic stem cell transplant pediatric recipients: a retrospective study. BMC Cancer 2016;16:590.

35. Poggio ED, McClelland RL, Blank KN, Hansen S, Bansal S, Bomback AS et al. Systematic review and meta-analysis of native kidney biopsy complications. Clin J Am Soc Nephrol 2020;15:1595 LP - 1602

36. Short SS, Papillon S, Hunter CJ, Stanley P, Kerkar N, Wang $\mathrm{L}$ et al. Percutaneous liver biopsy: pathologic diagnosis and complications in children. J Pediatr Gastroenterol Nutr 2013;57:644-648.

37. Younossi ZM. Non-alcoholic fatty liver disease-A global public health perspective. J Hepatol 2019;70:531-544.

38. Atar E, Ben Ari Z, Bachar GN, Amlinski Y, Neyman C, Knizhnik $\mathrm{M}$ et al. A comparison of transjugular and plugged-percutaneous liver biopsy in patients with contraindications to ordinary percutaneous liver biopsy and an "in-house" protocol for selecting the procedure of choice. Cardiovasc Intervent Radiol 2010;33:560-564.

39. Filingeri V, Francioso S, Sforza D, Santopaolo F, Oddi FM, Tisone G. A retrospective analysis of 1.011 percutaneous liver biopsies performed in patients with liver transplantation or liver disease: ultrasonography can reduce complications? Eur Rev Med Pharmacol Sci 2016;20:3609-3617.

40. Hall TC, Deakin C, Atwal GS, Singh RK. Adequacy of percutaneous non-targeted liver biopsy under real-time ultrasound guidance when comparing the Biopince ${ }^{\mathrm{TM}}$ and Achieve ${ }^{\mathrm{TM}}$ biopsy needle. Br J Radiol 2017;90:20170397.

41. Hu C-T. Co-axial liver biopsy is the solution for hepatologists to obtain adequate tissue for molecular studies. Hepatol Int 2010;4:342.

42. Kim SJ, Won JH, Kim YB, Wang HJ, Kim BW, Kim H et al. Plugged percutaneous biopsy of the liver in living-donor liver transplantation recipients suspected to have graft rejection. Acta Radiol 2017;58:771-777.

43. Mogahed EA, Mansy YA, Al Hawi Y, El-Sayed R, El-Raziky $\mathrm{M}$, El-Karaksy H. Blind percutaneous liver biopsy in infants and children: Comparison of safety and efficacy of percussion technique and ultrasound assisted technique. Arab J Gastroenterol 2016;17:168-175.

44. Procopet B, Bureau C, Métivier S, Selves J, Robic MA, Christol $\mathrm{C}$ et al. Tolerance of liver biopsy in a tertiary care center: comparison of the percutaneous and the transvenous route in 143 
prospectively followed patients. Eur J Gastroenterol Hepatol 2012;24:1209-1213

45. Sandrasegaran K, Thayalan N, Thavanesan R, Kohli M, Berry W, Shah A et al. Risk factors for bleeding after liver biopsy. Abdom Radiol 2016;41:643-649.

46. Seeff LB, Everson GT, Morgan TR, Curto TM, Lee WM, Ghany $\mathrm{MG}$ et al. Complication rate of percutaneous liver biopsies among persons with advanced chronic liver disease in the HALT-C trial. Clin Gastroenterol Hepatol 2010;8:877-883.

47. West J, Card TR. Reduced mortality rates following elective percutaneous liver biopsies. Gastroenterology 2010;139:1230-1237.

48. Atwell TD, Smith RL, Hesley GK, Callstrom MR, Schleck $\mathrm{CD}$, Harmsen WS et al. Incidence of bleeding after 15,181 percutaneous biopsies and the role of aspirin. Am J Roentgenol 2010;194:784-789.

49. Bilreiro C, Noruegas MJ, Gonçalves I, Moreira Â. Ultrasoundguided liver biopsies in children: A single-center experience and risk factors for minor bleeding. J Pediatr Gastroenterol Nutr. 2017;65:137-140

50. Matos H, Noruegas MJ, Gonçalves I, Sanches C. Effectiveness and safety of ultrasound-guided percutaneous liver biopsy in children. Pediatr Radiol 2012;42:1322-1325.

51. Nodarse-Pérez PO, Pérez-Menéndez R, Heredia-Andrade ED, Noa-Pedroso G, Araluce-Cordoví R, Fernández-Sotolongo J. Seguridad de la reducción del tiempo de reposo posbiopsia hepática percutánea y por vía laparoscópica. Surg Surg 2016;84:196-202.

\section{Authors and Affiliations}

52. Pan A, Alansari M, Lubcke R, Schlup M, Williams M, Fraser $\mathrm{M}$ et al. Use of pethidine for percutaneous liver biopsy-a randomised, placebo-controlled, double blind study. BMC Gastroenterol 2015;15:33.

53. Sornsakrin M, Helmke K, Briem-Richter A, Ganschow R. Value of ultrasound-guided percutaneous liver biopsy in children following liver transplantation. J Pediatr Gastroenterol Nutr 2010;51:635.

54. Vijayaraghavan GR, Vedantham S, Rangan V, Karam A, Zheng L, Roychowdhury A et al. Effect of needle gauge and lobe laterality on parenchymal liver biopsy outcome: a retrospective analysis. Abdom Imaging 2015;40:1223-1229.

55. Westheim BH, Aagenæs I, Østensen AB, Sanengen T, Lmaas R. Effect of operator experience and frequency of procedure performance on complication rate after ultrasound-guided percutaneous liver biopsies. J Pediatr Gastroenterol Nutr 2013;57:638-643.

Publisher's Note Springer Nature remains neutral with regard to jurisdictional claims in published maps and institutional affiliations.

\section{Helena B. Thomaides-Brears ${ }^{1}$ (D) Naim Alkhouri ${ }^{2} \cdot$ Daniela Allende $^{3,4} \cdot$ Mukesh Harisinghani $^{5} \cdot$ Mazen Noureddin $^{6}$. Nancy S. Reau ${ }^{7} \cdot$ Marika French $^{1}$ - Carlos Pantoja ${ }^{8} \cdot$ Sofia Mouchti ${ }^{1}$ - Donna R. H. Cryer ${ }^{4}$}

Naim Alkhouri

naim.alkhouri@gmail.com

Daniela Allende

allendd@ccf.org

Mukesh Harisinghani

MHARISINGHANI@MGH.HARVARD.EDU

Mazen Noureddin

Mazen.Noureddin@cshs.org

Nancy S. Reau

Nancy_Reau@rush.edu

Marika French

marika.french@perspectum.com

Carlos Pantoja

Carlos.Pantoja@perspectum.com

Sofia Mouchti

Sofia.Mouchti@perspectum.com

Donna R. H. Cryer

dcryer@globalliver.org
1 Perspectum, Gemini One, 5520 John Smith Drive, Oxford OX4 2LL, UK

2 Arizona Liver Health, Phoenix, AZ, USA

3 Pathology Department, Cleveland Clinic, Cleveland, $\mathrm{OH}$, USA

4 Global Liver Institute, Washington, USA

5 Department of Radiology, Massachusetts General Hospital, Harvard Medical School, Boston, USA

6 Division of Digestive and Liver Diseases, Comprehensive Transplant Center, Cedar Sinai Medical Center, Los Angeles, CA, USA

7 Department of Internal Medicine, Division of Digestive Diseases and Nutrition, Rush Medical College, Chicago, USA

8 South San Francisco, Perspectum, CA, USA 